\title{
Real-time interpolation of global ionospheric maps by means of sparse representation
}

\author{
Heng Yang ${ }^{1,2,3} \cdot$ Enric Monte-Moreno ${ }^{1}\left[\right.$ - Manuel Hernández-Pajares ${ }^{3} \cdot$ David Roma-Dollase $^{4}$
}

Received: 2 December 2020 / Accepted: 28 May 2021 / Published online: 12 June 2021

(c) Springer-Verlag GmbH Germany, part of Springer Nature 2021

\begin{abstract}
In this paper, we propose a method for the generation of real-time global ionospheric map (RT-GIM) of vertical total electron content (VTEC) from GNSS measurements. The need for interpolation arises from the fact that the ionospheric pierce point (IPP) measurements from satellites to stations are not distributed uniformly over the ionosphere, leaving unfilled gaps at oceans or poles. The method we propose is based on using a high-quality historical database of post-processed GIMs that comprises more than two solar cycles, calculates the GIM by weighted superposition on a subset of the database with the compatible solar condition. The linear combination of GIMs in the database was obtained by minimizing a $\ell_{2}$ distance between VTEC measurements at the IPPs and the VTECs from the database, adding a $\ell_{1}$ penalization on the weights to assure a sparse solution. The process uses a Sun-fixed geomagnetic reference frame. This method uses the atomic decomposition/least absolute shrinkage and selection operator (LASSO), which will be denoted as atomic decomposition interpolator of GIMs (ADIGIM). As the computation is done in milliseconds, the interpolation is performed in real time. In this work, two products were developed, denoted as UADG and UARG, the UADG in real time and UARG with a latency of $24 \mathrm{~h}$ to benefit from the availability of a greater number of stations. The altimeter JASON3 VTEC measurements were used as reference. The quality of interpolated RT-GIMs from day 258 of 2019 to 155 of the year 2020 is compared with other RT/non-RT GIM products such as those from International GNSS Service (IGS), Centre National d'Etudes Spatiales (CNES), Chinese Academy of Sciences (CAS), Polytechnic University of Catalonia (UPC) and others. The RT ADIGIM performance proved to be better, nearly as good as the rapid or final GIMs computed retrospectively with delays of hours to days. Besides, the non-RT ADIGIM quality is as good or better than most GIM products. The oceanic regions have been included in the assessment which showed that ADIGIM interpolation gives the best estimation (referred to JASON3). The developed method, UADG, will constitute the next-generation UPC RT-GIM, and also UARG will improve the current product UQRG (the current UPC rapid GIM product computed retrospectively) due to its complementary information.
\end{abstract}

Keywords Real-time global ionospheric map (RT-GIM) - Atomic decomposition interpolator of GIMs (ADIGIM) - Sparse representation · Vertical total electron content (VTEC) · Global navigation satellite system (GNSS)

Enric Monte-Moreno

enric.monte@upc.edu

1 Department of Signal Theory and Communications, TALP, Universitat Politècnica de Catalunya, 08034 Barcelona, Spain

2 School of Electronic Information and Engineering, Yangtze Normal University, Chongqing 408100, China

3 Department of Applied Mathematics IV, IonSAT, Universitat Politècnica de Catalunya, 08034 Barcelona, Spain

4 IEEC-CSIC, Campus UAB, Carrer de Can Magrans s/n, 08193 Cerdanyola del Vallès, Spain

\section{Introduction}

The global ionospheric maps (GIMs) are defined as a twodimensional array, where the elements represent the vertical total electron content (VTEC) mapped to a single-layer ionospheric shell at an effective height, corresponding to a partition in rectangles defined according to latitude and longitude divisions. Typically, the value of the VTEC at each grid point is determined from a permanent global network of the global navigation satellite systems (GNSS) by performing multi-frequency measurements for the ionospheric delay on the line of sight from satellite to receiver, and modeling it to the vertical component (Schaer 1999). The importance 
of having a good real-time (RT) estimate of GIM is that they play an important role in applications such as improving precise navigation, monitoring footprints of natural hazards, and others (see for instance Lin 2010; Hernández-Pajares and Roma-Dollase 2017).

Under the umbrella of the International GNSS Service (IGS) (Dow et al. 2009), several ionosphere-associated analysis centers (IAACs) around the world contribute to the calculation of GIM products by using different techniques. The products provided have been independently available for the last few decades (since 1998) and have demonstrated to be reliable (Hernández-Pajares et al. 2009). Providers of this service are the following organizations, the Center for Orbit Determination in Europe (CODE) (Schaer et al. 1996), Jet Propulsion Laboratory (JPL) (Mannucci et al. 1998), Polytechnic University of Catalonia (UPC) (Hernández-Pajares et al. 1999), European Space Agency (ESA) (Feltens 2007), Wuhan University (WHU) (Zhang et al. 2013), Natural Resources Canada (NRCan) (Ghoddousi-Fard 2014), Chinese Academy of Sciences (CAS) (Li et al. 2015), and in particular, the IGS final product, IGSG, as the weighted combination of above-mentioned GIMs (Hernández-Pajares et al. 2009, 2018; Roma-Dollase et al. 2018).

Thanks to the availability of IGS's GNSS RT service, the GNSS observations are accessible from the worldwide datastreams in real time with a latency of few seconds (Caissy and Agrotis 2011). Using the flow of GNSS observations, several specialized centers that work on ionospheric models provide GIMs in real time. Among the centers that provide this information, we can mention the UPC (Roma Dollase et al. 2015), CAS (Wang and Li 2018), Centre National d'Etudes Spatiales (CNES) (Laurichesse and Blot 2015) and German Aerospace Center in Neustrelitz (DLR-NZ) (Jakowski et al. 2011) and WHU (Ren et al. 2019), among others.

The distribution of observations from GNSS is sparse and unevenly distributed with different geographical densities. Besides, it depends on the underlying geography, so that ocean, desert, and polar areas are not well covered in terms of receivers. To obtain estimates of VTEC values in areas that are not well covered by the GNSS receiver network, several techniques have been developed for interpolating VTEC values such as spherical harmonics (Schaer 1999; Feltens 2007), integrated using the generalized trigonometric series functions ( $\mathrm{Li}$ et al. 2015) and with the inequality-constrained least squares (Zhang et al. 2013), using a three-shell model with bi-linear spline interpolation (Mannucci et al. 1998), tomography with splines (Hernández-Pajares et al. 1999), and tomography with Kriging (Orús et al. 2005). These techniques, applied to the post-processed GNSS observation data, have provided excellent estimates for the global state of the ionosphere, with less than $30 \%$ relative errors and 3.6-5.3 TECUs $\left(1 \mathrm{TECU}=10^{16} \mathrm{el} / \mathrm{m}^{2} \approx 16 \mathrm{~cm}\right.$ delay in L1 GPS signal) of standard deviation, according to a long-term validation by different approaches (Roma-Dollase et al. 2018).

Nevertheless, the RT-GIMs estimates give a worse performance compared with the ionospheric model of GIMs computed retrospectively with a delay of hours to days (e.g., the rapid vs. final products). The worsening of the performance in the RT estimate is due to the non-uniformity and sparseness of RT broadcasters, the time-varying quality of RT data processing in addressing the estimation of the ambiguity of the ionospheric combination of carrier phases, and unforeseen issues in RT processing. Finally, there is a compromise between spatio-temporal resolution and computation complexity.

In particular, the approaches based on spherical harmonics and Kriging provide an excellent representation of global VTEC in the post-processed products but have been proven to perform worse in RT ionospheric modeling. This is not only due to the lack of available receivers in real time but it is especially motivated for the lack of ambiguity convergence in real time for most ionospheric combinations of carrier phases. According to the precedent VTEC assessments, the quality of the RT-GIMs, such as CAS, CNES, and, UPC, is approximately $8.6-22.5 \%$ worse than the post-processed products (Hernández-Pajares and Roma-Dollase 2017; Li et al. 2020).

In this work, we introduce an interpolation technique, denoted as Atomic Decomposition Interpolator of GIMs (ADIGIM). The ADIGIM technique allows for RT calculation of high-quality GIMs, especially in the case of noisy GNSS observations and with non-uniformly distributed observations. As the computation time is of few seconds in a standard desktop computer, the maps are generated in real time, i.e., at the rate of VTEC measurements obtained from Ionospheric Pierce Points (IPPs). The ADIGIM algorithm approximates these IPPs measurements from a sparse linear combination of high-quality GIMs from a historical database. This sparse approximation from the historical database provides additional information on the dates when the ionospheric maps were most similar to the current measurements.

The paper is structured as follows: First, we discuss the UPC RT Tomographic Ionospheric Model (UPC RTTOMION) for GNSS data processing, then we present the methodology of interpolation using the ADIGIM algorithm, and the performance and robustness assessment.

\section{UPC real-time VTEC data stream}

The input data for the RT interpolation of GIMs rely on several hundred GNSS stations distributed around the world. The flow of data is accessed via the data streaming protocol used so far by the RT IGS working group and processed by UPC-IonSAT's ionosphere model. 
The streaming protocol referred to as "Networked Transport of Radio Technical Commission for Maritime Services (RTCM) via Internet Protocol" (NTRIP), was developed by the German Federal Agency for Cartography and Geodesy (BKG), enables the streaming of the observation data from the worldwide permanent GNSS receivers (Weber et al. 2005).

The UPC-IonSAT's RT TOMographic IONosphere Model (RT-TOMION) is a 4D (3D+time) model of the global state of the ionosphere, focused on RT estimation of total electron content modeling, mainly based on GPS dual-frequency measurements with the hybrid geodetic and tomographic ionospheric model, and robust to various types of degradation.

This model is the evolution of the Tomographic Ionospheric Model (TOMION) developed by UPC in the 1990s and has been employed for UPC RT/near-RT ionosphere service of IGS since 2011 (Hernández-Pajares et al. 1999, 2000; Orús et al. 2005; Hernández-Pajares 2014; Roma Dollase et al. 2015).

The RT-TOMION model avoids the distortion of singlelayer GIM, by decomposing the ionosphere as a two-layer structure framed by voxels in an almost Sun-fixed reference frame where the electron density in each is assumed to be a slowly varying Random Walk process and estimated at the same time that the carrier phase ambiguities are estimated from the contained lines of sight. The RT process of the GNSS data flow is organized in the following steps:

- Collect dual-frequency carrier phase data $L_{1}$ and $L_{2}$ from GNSS data streaming, solve the carrier-phase ionospheric ambiguity $B_{I}$, and estimate the slant total electron content (STEC) affecting GNSS signals as $L_{I}=L_{1}-$ $L_{2} \approx \alpha_{I} S T E C+B_{I}$ (Hernández-Pajares et al. 1999), where $\alpha_{i}=40.3 / f_{i}^{2}$.

- Partition the ionosphere into voxels with a dedicated spatial resolution and describe the global tomographic electronic density from multiple-data flow of ground GNSS stations by the Kalman filter solution, together with the satellite and receiver differential code biases (DCBs).

- Generate the single-layer VTEC map at an assigned effective height (e.g., 450km), simplified from the global tomographic electronic density model, feed for interpolating the map of global VTEC values of IPPs as the GIMs.

Also, the VTEC interpolation techniques of the UPC RTTOMION model carried out either by spherical harmonics or Kriging (Orús et al. 2005) is used to fill the gaps where there is a lack of data. This lack of data is due to the inhomogeneous distribution and sparsity of GNSS receivers (Feltens et al. 2011; Hernández-Pajares et al. 2016).
As an illustration for understanding the VTEC maps interpolating for GIMs presented in this paper, Fig. 1 shows the processed VTEC data by UPC RT-TOMION model, consisting of RT VTEC calculations of selected GPS stations during the interval of 13:15-13:30 UTC, on day 1 of 2020. Note that the VTEC map (see Fig. 1B) shows the noise in the RT solution for carrier-phase ionospheric ambiguity, which can significantly degrade the GIM quality, regardless of whether the interpolation technique used is spherical harmonics or Kriging.

\section{GIM interpolation by sparse representation}

\subsection{Motivation for the interpolation algorithm}

The interpolation method that we propose is based on the work carried out by the authors related to the estimation of Traveling Ionospheric Disturbances (TIDs) in the ionosphere (Yang et al. 2017; Yang 2019). This method is inspired by the work of Wright et al. $(2008,2010)$ in the context of face recognition.

It should be noted, however, that the problem of estimating TIDs is different from the one we deal with in this paper. The difference is in the sense that the previous work consists of determining the number of TIDs present, together with the characterization in terms of velocity, wavelength, amplitude, and phase. In other words, it is a dynamic problem. It should be noted that the fact that the number of TIDs is not known introduces a special difficulty to the problem. On the other hand, in this paper, we try to determine a linear combination of previously measured GIM maps that best approximate the measurements made on a set of ground stations. And especially address the problem of areas without station coverage. The problem is static in that it attempts to represent current measurements as a function of global maps. The mathematical common point between the two methods is that they try to minimize a cost function with an L2 criterion, with an L1 regularization term that introduces sparsity.

The following analogy with the problem of face recognition may allow us to understand the properties of the method we use to perform the interpolation,

- In Wright et al. (2008, 2010), the authors create a database with the faces of all possible individuals, while in our case we create a database with the high-quality GIMs in all the conditions of the ionosphere and solar climatology during the last two solar cycles (24 years).

- The algorithm has to select a small subset of faces, which matches the values of the test face. In our case, we will select a subset of the historical GIMs that will match the GNSS IPP measurements at the current moment. 


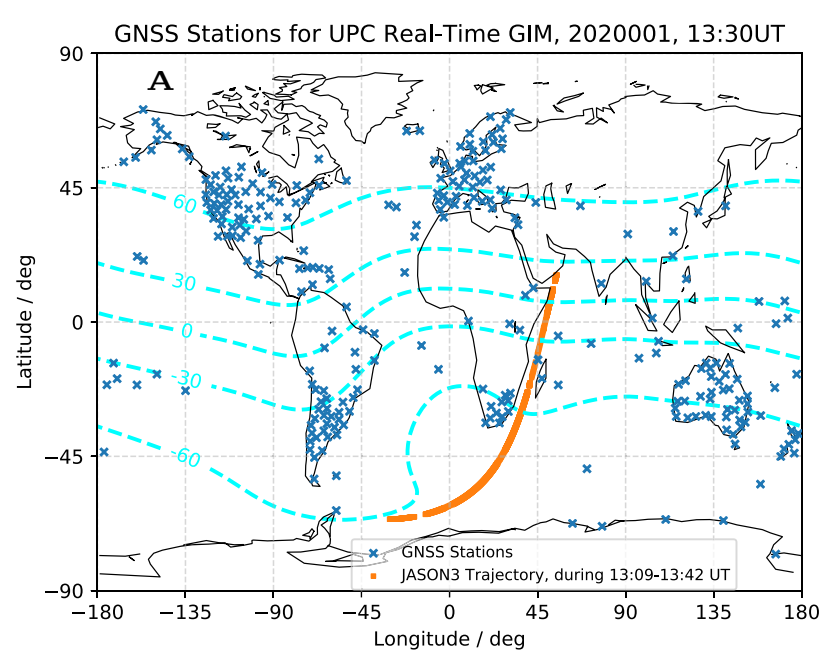

Fig. 1 a Selected RT ground GNSS stations marked in blue, at 13:30 UT of January 1, 2020. The orange dots show the trajectory of altimeter JASON3 from 13:09-13:42 UT; b the RT VTEC map composed of IPPs

- The face recognition system has to be robust to occlusions (use of glasses), changes of hairstyle. In our analogy would correspond to variation in the number of stations and noise measurements (e.g., by low satellite elevation) and regions not covered by stations (see Fig. 1B).

The interpolation solution we propose consists of adapting the solution found for the problem of estimation of wavefronts (such as Yang et al. 2017; Yang 2019) into a problem of gap filling. That is, we will state the problem of estimating TIDs from a dictionary of possible waveforms as an interpolation problem from a historical dictionary of GIMs. The key point is finding the elements of the dictionary that match the current IPP measurements. Unlike the TIDs dictionary (Yang 2019), where the reference database consisted of wavefronts, with combinations of amplitude, frequency, phase, and orientation that covered the possible realistic TIDs compatible with the pierce point measurements, in this work the reference dictionary is a historical set of high-quality GIMs, and the detection problem is transformed into a combination of GIMs compatible with the current IPP measures. Thus, the interpolation over regions not covered by the satellite/stations is done by using the past examples of interpolated GIMs similar to the current measures at the pierce point locations. In this work, the UPC rapid GIM (UQRG) is to be used as the past GIMs due to its good quality, which is computed by the tomography approach connected with ordinary Kriging interpolation.

An example of the set of measured IPPs used for creating the RT-GIM estimates with a 15-min resolution is shown in Fig. 1B. The VTEC map consists of IPPs measured by the RTTOMION model in a 15 minutes interval. The figure clearly

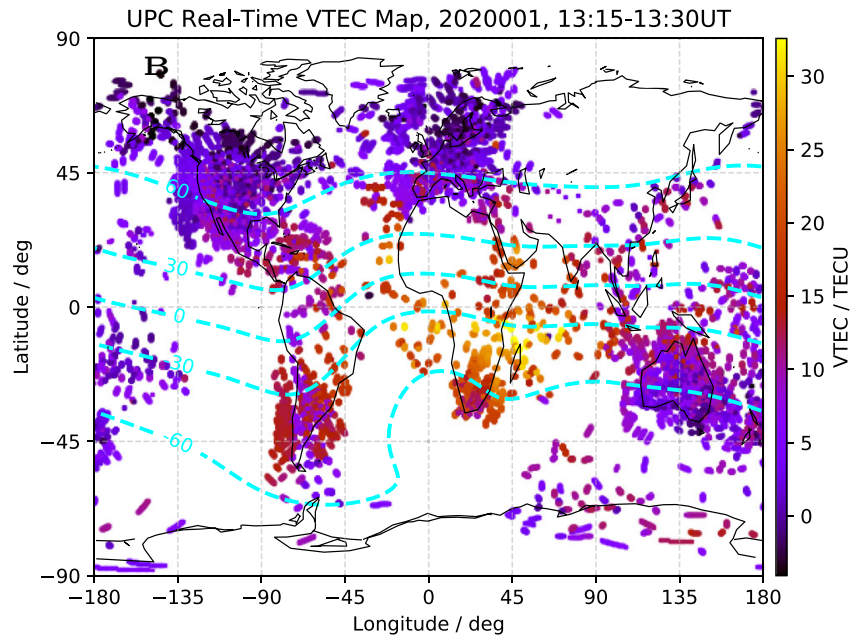

and associated VTEC during 13:15-13:30 UT. The light cyan dashed lines for the geomagnetic dip angles, see Thébault et al. (2015)

shows that there are regions of the Earth that are not covered by IPPs. Note that a measurement longer than the temporal resolution would increase the global coverage of IPPs at the price of mixing past ionospheric information that would not reliably represent the ionospheric state at the current moment. This is the case, for instance, the last $1-2 \mathrm{~h}$ of VTEC data interpolating for UPC RT-GIM with 15-min resolution (Orús et al. 2005), and the last 15 min of VTEC data for CAS RTGIM with 5-min resolution (Li et al. 2020). Another related consideration is that the presented interpolation technique is evaluated within the VTEC map of IPPs measured by GPS without additional IPPs of multi-GNSS to show the algorithm performance in a typical condition.

The distribution and density of IPPs are not uniform on the surface of the earth, leaving areas uncovered, and since the VTEC estimation from the IPPs will depend on the inclination of the satellites and the length of continuous arc phase. We will also have an uneven distribution of the magnitude of the estimation noise. The difference in the relative size of the noise on the IPPs can be seen in Fig. 1B, as local variability in the size (color-coded) of the VTEC. To specify the interpolation model, we construct the VTEC map of GPS measurements from the UPC RT-TOMION data stream and define the IPPs at the conventional IGS effective height (e.g., $450 \mathrm{~km}$ ) of the single-layer model as shown in Fig. 1B.

The ionospheric map is represented in Sun-fixed geomagnetic coordinates. This is because the morphology of the ionosphere depends on the geographical location of the Sun and the geomagnetic distribution. Using Sun magnetic coordinates will guarantee that the peak of ionization will be located around the center of the map. This is done using the equidistant cylindrical projection these coordinates are 
transformed into planar coordinates, i.e., $(x, y)$ directly representing Sun-fixed geomagnetic longitude and latitude. The model of the measurements is a linear combination of Kronecker deltas located at the set of IPP planar coordinates, that is

$$
V(x, y)=\sum_{\left(x_{i}, y_{j}\right) \in I_{x, y}} A_{i, j} \delta\left(x-x_{i}, y-y_{j}\right)
$$

where $I_{x, y}$, is the set of IPP coordinates $\left(x_{i}, y_{j}\right)$ at measurement interval $t$, i.e., the coordinates at effective height of the line-of-sights that join GNSS stations and satellites, and $A_{i, j}$ is the estimated VTEC $V(x, y)$ at coordinates $\left(x_{i}, y_{j}\right)$ in the set $I_{x, y}$.

The underlying assumption is that the approximate global profile of ionospheric electron content tends to repeat cyclically, depending on the time of day, season, and solar activity. This dependence of the ionosphere has been investigated and confirmed, according to the previous case studies of ionospheric variability for more than two solar cycles, see details in Balan et al. (1996), Forbes et al. (2000) and Solomon et al. (2013). Although the solar non-ordinary activity has non-cyclic components, such as solar flares among geomagnetic storms, we assume that the spatial variability of ionization can be modeled as a linear combination of a few previous GIMs associated with similar solar conditions. Salinas et al. (2016) and Dmitriev et al. (2017) investigate and study historical geomagnetic storm events in ways of different observing approaches and physical models, where the consistent characteristics of regional ionospheric responses confirm the feasibility of the non-cyclic component model. Therefore, the GIMs that we use to estimate ionospheric state at a given moment will be the retrospectively calculated maps that correspond to the comparable conditions.

The technique for solving the above-stated problem is based on two techniques of sparse reconstruction, i.e., the atomic decomposition (Chen et al. 2001) and the Least Absolute Shrinkage and Selection Operator (LASSO) (Hastie et al. 2015). These techniques are based on finding a solution to a minimization problem that is a combination of an approximation error by norm $\ell_{2}$, along with a $\ell_{1}$ penalization on the approximation coefficients. The $\ell_{1}$ penalization on the approximation coefficients induces sparsity. That is, only a small subset of the elements of the dictionary used for the approximation will be active. Thus, the interpolation is reduced to solving a convex optimization problem with a regularization term.

The model consists of a dictionary of a few past GIMs in which the elements are represented as $G_{t_{k}}$, i.e., the GIM of the historical database at epoch $t_{k}$. These $G_{t_{k}}$ are concatenated into the dictionary of past GIMs with all global observations. In this model, we approximate the VTEC measurement $V(x, y)$ of IPPs at current epoch $t$ (see Eq. 1) by a weighted combination of the dictionary elements at these coordinates. The weight associated with the element at IPPs $G_{t_{k}}^{I}(x, y)$ is denoted as $\alpha_{k}$. Furthermore, we assume that the current observation can be expressed in terms of the $M$ elements of the dictionary, using the locations of IPPs at the maps. Thus, the VTEC map $V(x, y)$ can be expressed as,

$V(x, y)=\sum_{k=0}^{M} \alpha_{k} G_{t_{k}}^{I}(x, y)+n(x, y)$

where the term $n(x, y)$ is an additive random noise, which deals with the fact that our model is not a generative model that takes into account physical features of the current state of the ionosphere but an approximation method that assumes that the current state of the ionosphere has a similarity with GIMs observed in the past and this term takes into account the possible differences. Note that the IPP coordinates and the noise of IPP VTEC determine the local features of GIMs, which significantly affect quality of solution of the regression model (see Eq. 2) by non-regularization methods. Also note the model for $V(x, y)$ uses only local information of the dictionary, that is, the past values at the coordinate set $I_{x, y}$.

Finally, the interpolated GIM $G_{t}$ is the estimate by a linear combination of past complete GIMs where the weight $\alpha_{k}$ associated with each map is related to the similarity of the current map at the pierce points with the past observations. The linear combination is as follows,

$G_{t}=\sum_{k=0}^{M} \alpha_{k} G_{t_{k}}$.

\subsection{Description of atomic decomposition interpolator of GIMs (ADIGIM)}

In this section, we will present a detailed description of the sparse interpolation algorithm for solving the proposed model in Sect. 3.1, the Atomic Decomposition Interpolator of GIMs (ADIGIM), outlined as a flowchart shown in Fig. 2. The first part of the algorithm consists on defining the dictionary $D$ of all the available historical GIMs. The dictionary $D$ is constructed by the concatenation of the set of $N$ past GIMs $G_{t_{k}}$ with $k=0,1, \ldots, N$, e.g., $800,000+$ instances of UPC rapid GIM (UQRG) covering last 24 years. The dictionary is a matrix $D$ defined as,

$D=\left[G_{t_{1}}, G_{t_{2}}, G_{t_{3}}, \ldots, G_{t_{N}}\right]$

For the interpolation at a given timestamp $t$, the dictionary is constructed using only elements of the complete historical database within the subset of assigned time intervals which must comply with conditions related to the season of the year and current solar activity, e.g., a union of 5 years centered on 
the current year in a solar cycle, 30 days on the current day in a year, and $2 \mathrm{~h}$ on the current hour in a day. The dictionary for the GIM at epoch $t$ is denoted as $D_{t}$, see Fig. 2. It is worth noting that the convex optimization problem is solved only for the coordinates corresponding to IPPs. The VTEC on the historical GIMs $G_{t_{k}}^{I}(x, y)$ at the IPPs are computed from VTEC of GIM grids using the bi-linear interpolation (Schaer et al. 1998).

Subsequently, for each of the GIM the set of VTEC values at the IPPs $G_{t_{k}}^{I}(x, y)$ are reshaped as vectors. That is, the measured VTEC is reshaped from the sparse 2D matrix (i.e, each non-zero element corresponds to an IPP) to a dense vector of dimension $P_{I}$, which is the number of IPP measurements. To avoid overloading the notation the new representation of the historical GIM VTEC will also be denoted as $G_{t_{k}}^{I}(x, y)$. Thus, we will construct a resulting dictionary as an array $D_{t}^{I}$ of dimension $\left(P_{I} \times N_{t}\right)$ with the $G_{t_{k}}^{I}(x, y)$ values extracted from the dictionary $D_{t}$.

The number of effective IPPs was reduced by a local average, which also reduced measurement noise which mainly contains the carrier phase ambiguity residuals in the realtime scenario, see Fig. 1B. Using all the IPPs improves the quality of the estimated maps but leads to an increase in the computational resources to estimate the GIMs. In this work, we opted for a solution that gave acceptable results in terms of the compromise between calculation quality and expense. The chosen solution consisted of averaging the VTEC values at the IPPs on a grid weighted by the distance to the center of masses and assigning the average value to the position of the center. The grid size is 2.5 degrees of latitude and 5 degrees of longitude, which is the same with GIM. On the other hand, since IPP measurements are noisy due to different satellite line-of-sight elevations and quality of carrier phase ambiguity, this average provides more reliable values. Associated to each element of the dictionary, we will define a vector of unknowns $\alpha_{t}$ of size $N_{t}$, which is the weight that is given in the solution to each $G_{t_{k}}^{I}(x, y)$. Hence, the estimation of the vector, $\alpha_{t}$, will only use the rows of $D_{t}^{I}$, such that the observation $V(x, y)$ in Eq. 2, is approximated by the product

$V(x, y) \approx D_{t}^{I} \alpha_{t}$.

As the observation vector $V(x, y)$ is defined by the values observed at the IPPs of each satellite, the dictionary matrix $D_{t}^{I}$ contains only the components at the observed IPPs. The parameter vector $\tilde{\alpha}_{t}$ is estimated by means of a trade-off between a loss function (i.e., how well we approximate the observations at IPPs) and a regularization term (i.e., constraints on the possible solutions). The regularization term is used to guarantee that the solution should be sparse, i.e., only a small set of elements of the dictionary will contribute to the approximation. The sense of a sparse solution lies in the property reported in Wright et al. (2010, 2008), which allows us to account for the occlusions in images, which in our case corresponds to the areas not covered by IPPs. An estimation technique that allows for the trade-off with a sparse set of coefficients is the LASSO (see for instance Hastie et al. 2015), where the norm on the loss function is $\ell_{2}$, and the norm on the regularization term is $\ell_{1}$. Thus, the optimization problem to be solved is,

$\tilde{\alpha}_{t}=\arg \min _{\alpha_{t}} \frac{1}{2}\left\|V-D_{t} \alpha_{t}\right\|_{\ell_{2}}+\rho\left\|\alpha_{t} \cdot\right\|_{\ell_{1}}$

The parameter $\rho$ controls the level of the sparsity of the solution and is determined automatically from the data, as explained in (Yang et al. 2017). The intuition regarding this parameter is related in that it makes a compromise between the approximation error (first term), and the sum of the absolute value of the weights (second term). When $\rho$ is equal to zero, the problem is reduced to a simple least squares approximation. On the other hand, since the penalty is in norm 1, the small non-zero values count and penalize, which leads to preferring solutions in which the number of non-zero weights is less than what we would have in the quadratic case (norm 2). Finally, the interpolated GIM is computed as with the dictionary $D_{t}$ (i.e., the historical GIMs within epochs $T_{t}$, using all grids), as follows:

$G_{t}=D_{t} \tilde{\alpha}_{t}$

Thus, the map $G_{t}$ is a complete map generated from a sparse linear combination of historical GIMs from the database $D_{t}$. As complete maps are used, the linear combination not only computes the VTEC from grids of adjacent to IPPs but also fills the gaps corresponding to regions with no IPP and thus makes an interpolation using values of a subset of past maps implicitly assuming a sort of ergodicity. Besides, the minimization of the cost function (see Eq. 6) was done on the interpolated coordinates that correspond to IPPs. As the dictionary elements (GIMs) were estimated at different times of the day/month/solar cycle, the sets of IPPs of the complete maps that fill the gaps are likely to correspond to good estimates in terms of the past ionospheric state from reliable GIMs.

Another interesting byproduct of the method is that the vector $\alpha_{t}$ selected and weights the past maps that are more similar to the current observed measurements might be used to explain features of the current map by comparing information available for the selected past maps. In other words, they might constitute the baseline of a GIM-based VTEC climatic model. 

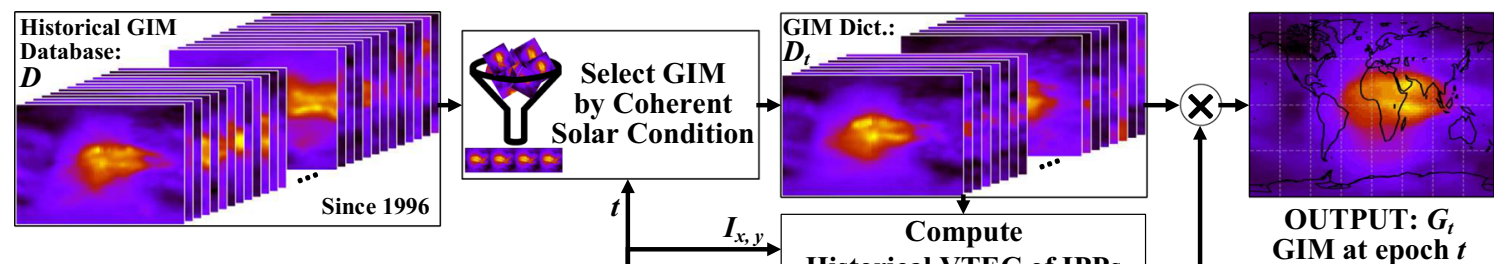

GIM at epoch $t$

INPUT: $V(x, y)$

VTEC map at epoch
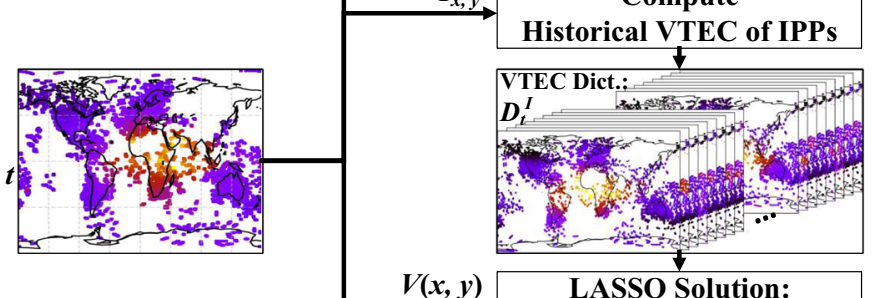

LASSO Solution:

$V=D_{t}^{I} \cdot a_{t}+n$

Fig. 2 Flowchart of ADIGIM: Given the RT set of measurements $V(x, y)$ expressed by IPP locations $I_{x, y}$ at epoch $t$, using maps of the historical GIM database $D$ to select the subset $D_{t}$ with the similar solar

\section{Quality assessment by means of JASON3 VTEC measurements}

The natural benchmark for determining the quality of the interpolated GIMs is to use a direct external measure of the VTEC. The reference data used is from the JASON3 altimeter satellite, where the interpolation plays a fundamental role due to the lack of permanent GNSS receivers, i.e., over the oceans. The VTEC reference was gathered from the vertical phase ionospheric delay directly measured by dual-frequency altimeter satellites, which corresponds to a different approach compared to the measurement done by GNSS ionospheric sounding.

The importance of the measures obtained by JASON3 lies in the fact that it provides us with an objective reference of the real value to be estimated through interpolation. The measures provided by JASON3, allow us to determine whether the estimate made by interpolation provides a correct value or introduces biases. As the orbit altitude of JASON3 is about $\sim 1300 \mathrm{~km}$, the altimeter can count almost all the VTEC of the ionospheric state above the ocean region. It is important to emphasize that over the ocean areas, large interpolating errors appear due to their far distance from GNSS ground stations. Therefore, the use of JASON3 VTEC measurement allows for a critical evaluation of the algorithms in adverse circumstances. In this work, the raw observations of the JASON3 VTEC were processed to reduce the measurement noise. The process carried out included the use of a temporal sliding window, removal of outliers, and so on, as explained in Hernández-Pajares et al. (2017) and Roma-Dollase et al. (2018).

An alternative method for obtaining objective VTEC model assessment is the dSTEC assessment. We do not use condition, the vector of coefficients $\alpha_{t}$ of the linear regression, are computed minimizing the error at $I_{x, y}$, then the estimate $\tilde{\alpha}_{t}$ (note sparseness) are used to combine with $D_{t}$, which yields the estimated GIM $G_{t}$

this method to validate the results for the following reasons. Typically, the JASON3 VTEC assessment is a validation method for GIMs only over the ocean region, so it may be appropriate to consider the complementary assessment for GIMs over the land region, namely the dSTEC assessment, which compares the difference between the observed STEC along the phase-continuous satellite-station arc and the calculated STEC from GIM, see details in Hernández-Pajares et al. (2017). However, the usage of altimeter VTEC measurements to assess GIMs has been proven to be a good external assessment procedure, consistent with other methods based on GNSS data (behaving similarly to the dSTEC test, Hernández-Pajares et al. (2017)) but independent from GNSS and globally distributed. These are the main reasons behind focusing on altimeter data, being the JASON3 the one available during the whole period of analysis, see the former studies that used JASON2, JASON1, and TOPEX altimeters.

Following, previous studies in the IGS GIMs accuracy, we have focused on an external and global assessment, both conditions fulfilled by the altimeter-based assessment. This is a suitable way for assessing the GNSS-based Global Ionospheric Maps, consistent with other ways of assessing like the dSTEC-GNSS approach previously commented, with also the advantage of being done in the most challenging regions, over the oceans. In addition, to fairly assess the GIM quality by dSTEC assessment, the selection of unused stations is highly important, given that the selected ones could hardly meet the condition of being equidistant from any other stations used for GIMs. Considering that with the rather unstable availability of RT GNSS casters, the dSTEC measurements might easily introduce biases into the quality assessment of GIMs, the dSTEC assessment method is discarded in this work. 


\section{Robustness analysis of ADIGIM}

In this section, we will compare the performance of the ADIGIM interpolation with Kriging using as reference the JASON3 VTEC measurements. The approaches for comparing the robustness are the following:

- Performance in Real-Time/Post-Processing Scenarios (Sect. 5.1), where we compare the performance of different interpolation methods in real-time or post-process.

- Interpolation performance in a GNSS Data Loss event (Sect. 5.2), where we compare the degradation in performance that the interpolation algorithms suffer in the event of loss of stations.

- Performance Comparison of ADIGIM GPS-only Interpolation with the Multi-GNSS Interpolation (Sect. 5.3).

\subsection{Performance in real-time/post-processing scenarios}

In this section, we will discuss the evaluation of the ADIGIM algorithm and compare its performance to other methods on equal terms. That is its robustness and consistency in RT and non-RT modes. The RT interpolation has a lower quality of the GIMs compared with the retrospective GIM (nonRT) mainly due to the lower number of available stations and unreliable solutions to phase ambiguities (see Sect. 1). Other factors affecting the comparison are that some products use different sets of stations and different types of single/multiple GNSS systems, while the data source for selected UPC GIM products are limited to GPS observations.

Therefore in this section, to guarantee comparisons on equal terms, the evaluation for ADIGIM in terms of standard deviation and relative error will be done comparing with spherical harmonics and Kriging. Thus, the comparisons will be ADIGIM versus spherical harmonics and Kriging, in RT and non-RT. The results use the same RT or non-RT individual VTEC data for calculating UPC current GIMs see Table 1.

We selected the GIMs computed for 40 days, i.e., from day 115 to 155 of 2020 . The test consisted of comparing the performance of ADIGIM, the spherical harmonics, and Kriging interpolation in RT and non-RT scenarios. And as benchmark standard, we used the data from the JASON3 VTEC measurements. The RT-GIMs are computed from the global GNSS data flow processed by the UPC RT-TOMION model, while the retrospective ones from the archived GNSS data processed by the UPC TOMION model. And Both TOMION and RT-TOMION models have worked well during the selected 40 days. Note the geographic distribution of non-RT GNSS data is much more uniform than the RT ones due to the availability with a delay of ten thousand global
non-RT GNSS stations versus hundreds of RT ones. These results are assessed by VTEC values that are independently measured by the altimeter satellite JASON3, see details in Sect. 4. The quality of GIMs represented in terms of standard deviation and relative error are summarized in Table 1, using $\sim 1.34$ million JASON3 observations.

The overall error related to the non-RT estimate is very similar in all cases. This is explained because the interpolation method that uses a dictionary of past maps on the IPP is using a much better quality of VTEC data compared with the RT one. In the case of the non-RT estimation, the standard deviation and relative error (referred to JASON3 measurements) differ by less than $1 \%$. On the other hand, in the RT case, the relative error of the ADIGIM is $26.9 \%$ lower than the one obtained by Kriging and a $8.9 \%$ lower error than the one obtained by spherical harmonics. This is explained by the fact that the ADIGIM uses for the interpolation process examples from past non-RT GIM maps, which are of much better quality and have the smallest euclidean distance to the current IPP measurements. The result is that the standard deviation and the relative error are lower than the other methods. Note that the relative errors in non-RT results seem to be larger than in RT ones, for instance in spherical harmonics and ADIGIM, respectively. These inconsistencies are associated with the performance of the GNSS preprocessing models (i.e., TOMION and RT-TOMION) in the scenarios of interest. Due to the different ionospheric measurement characteristics between the altimeter JASON3 and the GNSS, the relative errors in non-RT results are in fact more realistic than the relative errors in RT results, see further discussion in Sect. 8.1.1.

The standard deviations in RT results for Kriging and Spherical Harmonics give rise to standard deviation values, respectively, $29 \%$ and $10 \%$ higher than the post-processing results. While ADIGIM only increased by $3 \%$ and even the RT results for ADIGIM were slightly better than the postprocessing ones for spherical harmonics. ADIGIM exhibits almost the same performance as Kriging in non-RT conditions, and also the far better RT performance than other algorithms.

\subsection{Interpolation performance in a GNSS data loss event}

In this section, we show that the ADIGIM as an interpolation method is more robust than Kriging or spherical harmonics. The robustness is in the sense of lower degradation of performance in the event of loss of stations. A clue to this robustness lies in the fact that the other interpolation approaches fill the gaps between stations by a combination of the current observations while the ADIGIM looks in a historical dictionary of well interpolated examples and 
Table 1 Comparison of the quality of GIMs for the Kriging, Spherical Harmonics (SH), and ADIGIM, in ways of the standard deviation (SD) (in TECUs) and the relative error (in percentage), the reference is the JASON3 VTEC

measurement with the $\sim 1.34$ million common observations (40 days, within days from 115 to 155 of 2020)

\begin{tabular}{lllll}
\hline Input GNSS data & Pre-process model & Algorithm & SD & ERR\% \\
\hline GPS archive & TOMION & Kriging & 2.28 & 44.3 \\
& & SH & 2.38 & 45.6 \\
\multirow{2}{*}{ GPS data-stream } & RT-TOMION & ADIGIM & 2.28 & 44.4 \\
& & Kriging & 3.21 & 51.9 \\
& & SH & 2.63 & 41.6 \\
& & ADIGIM & 2.35 & 37.9
\end{tabular}

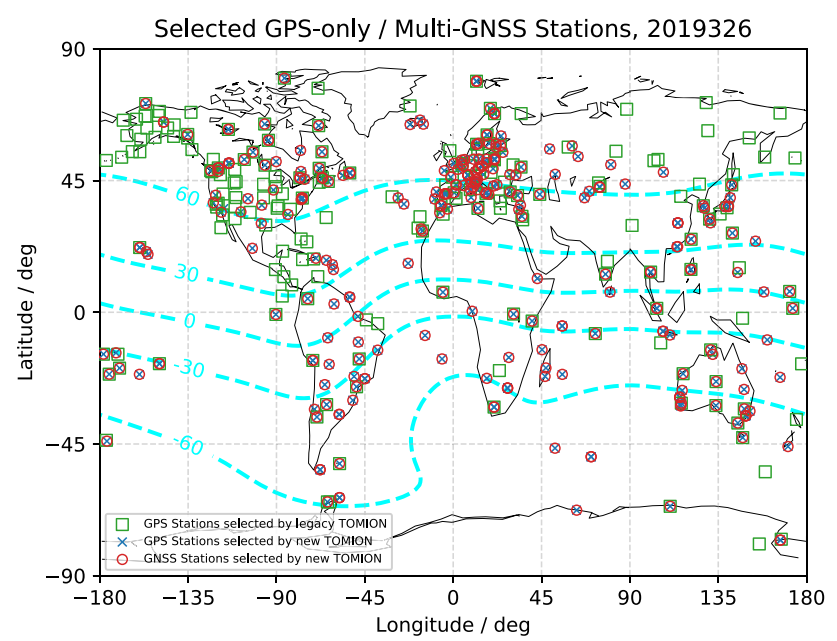

Fig. 3 Distribution of GNSS stations selected by TOMION models, for day 326 of 2019. The green rectangular is the GPS stations by the legacy TOMION model, the blue cross for GPS stations, and the red circle for Multi-GNSS stations of the experimental new TOMION model

selects the ones that are more similar to the current observed IPPs.

To illustrate this robustness to the loss of stations, we consider the day 326 of 2019, in a post-processing scenario, when the legacy UPC TOMION model for UQRG (Kriging interpolating) lost the connection to the archived data of greater than 30\% GNSS stations, in particular most significant ones deployed in the high latitude region of the southern hemisphere (illustrated as green rectangles in the map of Fig. 3). The result with the legacy model (i.e., uses archived Receiver INdependent EXchange format (RINEX) data in version 2) was that given identical input VTEC data, the standard deviation from Kriging interpolation (i.e., the legacy UQRG) at the latitudinal interval of $-50^{\circ}---70^{\circ}$ increased to 4.09 TECU nevertheless the ADIGIM GIM still kept at less than 3 TECU.

These results are summarized in Table 2, which show the local performance in bands of 20 degrees. The bands are a rough partition to better know the quality of the GIMs by implicitly removing from the latitudinal bias the signature of the electron content above the altimeter and below the GPS transmitter previously commented (and shown in Fig. 6). In this way, the standard deviation will reflect better the different performance of the GIM versus latitude. This is the compromise between the number of JASON3 passes in each band and the resolution of the ionosphere. As shown in bold, the relative error for the bands from $-30^{\circ}$ to -70 of the ADIGIM GIM changes less than 5\%, while the Kriging one is $18 \%$ higher. On the other hand, the performance for the rest of the latitudes is similar. Also note the geomagnetic activity was moderate with $k p \leq 4$ (GFZ-Postdam 2020), which might not create a significant disturbance of the ionosphere.
Table 2 GIM assessment compared to JASON3 VTEC, at 20-degree latitudinal bands (in TECUs), using GPS data

\begin{tabular}{|c|c|c|c|c|c|c|c|c|c|c|}
\hline \multirow[t]{3}{*}{ Lat $\left({ }^{\circ}\right)$} & \multicolumn{8}{|c|}{ With RINEX v2 GPS archive } & \multirow{2}{*}{\multicolumn{2}{|c|}{ JASON3 }} \\
\hline & \multicolumn{4}{|c|}{ Kriging } & \multicolumn{4}{|c|}{ ADIGIM } & & \\
\hline & Bias & SD & RMS & ERR\% & $\overline{\text { Bias }}$ & SD & RMS & ERR\% & $\overline{\mathrm{VTEC}}$ & \#OBS \\
\hline $50-70$ & -0.62 & 1.85 & 1.95 & 50.0 & -0.62 & 1.68 & 1.79 & 45.8 & 3.90 & 2771 \\
\hline $30-50$ & -2.10 & 1.83 & 2.79 & 38.0 & -1.41 & 1.90 & 2.37 & 32.3 & 7.33 & 4616 \\
\hline $10-30$ & -2.02 & 2.56 & 3.26 & 32.7 & -2.87 & 2.52 & 3.82 & 38.3 & 9.96 & 5745 \\
\hline-10 to 10 & -1.55 & 3.17 & 3.53 & 25.0 & -2.54 & 2.44 & 3.52 & 25.0 & 14.11 & 7034 \\
\hline-30 to -10 & -3.03 & 2.38 & 3.85 & 28.6 & -2.67 & 2.62 & 3.74 & 27.8 & 13.46 & 7557 \\
\hline-50 to -30 & -3.28 & 2.85 & 4.34 & 47.4 & -2.21 & 2.12 & 3.06 & 33.5 & 9.16 & 9880 \\
\hline-70 to -50 & -2.00 & 4.09 & 4.55 & 47.7 & -1.10 & 2.98 & 3.17 & 33.3 & 9.54 & 9070 \\
\hline
\end{tabular}

Left column: legacy TOMION model plus Kriging. Center: ADIGIM. Right: Jason3 reference, on day 326 of 2019 
Table 3 Summary of GIMs for quality comparison

\begin{tabular}{|c|c|c|c|c|}
\hline GIM Id & Agency & Type & Method & References \\
\hline IGSG & IGS & Final & Weighted Mean & Hernández-Pajares et al. (2009) \\
\hline CODG & CODE & Final & Spherical Harmonics (SH) & Schaer (1999) \\
\hline ESAG & ESA & Final & $\mathrm{SH}$ & Feltens (2007) \\
\hline JPLG & JPL & Final & Three-shell Model & Mannucci et al. (1998) \\
\hline UPCG & UPC & Final & Tomography with Splines & Hernández-Pajares et al. (1999) \\
\hline EMRG & NRCan & Final & SH & Ghoddousi-Fard (2014) \\
\hline CASG & CAS & Final & $\begin{array}{l}\text { SH and Generalized Trigonometric } \\
\text { Series }\end{array}$ & Li et al. (2015) \\
\hline WHUG & WHU & Final & $\begin{array}{l}\text { SH and Inequality- constrained } \\
\text { Least Squares }\end{array}$ & Zhang et al. (2013) \\
\hline CORG & CODE & Rapid & SH & Schaer (1999) \\
\hline ESRG & ESA & Rapid & SH & Feltens (2007) \\
\hline EHRG & ESA & Rapid & SH & Feltens (2007) \\
\hline JPRG & JPL & Rapid & Three-shell Model & Mannucci et al. (1998) \\
\hline UQRG & UPC & Rapid & Tomography with Kriging & Orús et al. (2005) \\
\hline CARG & CAS & Rapid & $\begin{array}{l}\text { SH and Generalized Trigonometric } \\
\text { Series }\end{array}$ & Li et al. (2015) \\
\hline WHRG & WHU & Rapid & $\begin{array}{l}\text { SH and Inequality-constrained } \\
\text { Least Squares }\end{array}$ & Zhang et al. (2013) \\
\hline CASR & CAS & RT & SH and Prediction Model & Li et al. (2020) \\
\hline CLK9 & CNES & RT & $\mathrm{SH}$ & Laurichesse and Blot (2015) \\
\hline $\mathrm{URTG}^{\mathrm{a}}$ & UPC & $\mathrm{RT}$ & Tomography with Kriging & Hernández-Pajares and Roma-Dollase (2017) \\
\hline USRG & UPC & RT & Tomography with SH & \\
\hline UARG & UPC & Rapid & Tomography with ADIGIM & Proposed in this work \\
\hline UADG & UPC & RT & Tomography with ADIGIM & Proposed in this work \\
\hline
\end{tabular}

List of agency acronyms: IGS for International GNSS Service, CODE for Center for Orbit Determination in Europe, ESA for European Space Agency, JPL for Jet Propulsion Laboratory, UPC for Polytechnic University of Catalonia, NRCan for Natural Resources Canada, CAS for Chinese Academy of Sciences, WHU for Wuhan University

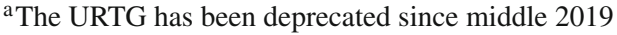

\subsection{Performance comparison of ADIGIM GPS-only interpolation with the multi-GNSS interpolation}

In this section, we will present the results of ADIGIM interpolation using a historical dictionary made up of maps interpolated only with GPS data and will compare it with maps generated from the interpolation using multi-GNSS data. Note that multi-GNSS data consist of a higher number of IPPs than the GPS only and that although the ADIGIM uses historical maps derived from GPS data, the IPPs will be equal to the ones of the methods with which it is compared. Also, note that in this case the comparison with the ADIGIM interpolation is done in disadvantaged because the dictionary consists of past maps interpolated using of Kriging using GPS data, i.e., a much lower number of IPPs used for the interpolation compared with the case of Kriging with multiGNSS.
The legacy TOMION model employed only GPS data and suffered the loss of available GPS archives with RINEX version 2. This justifies the use of the experimental new TOMION model designed for flexible RINEX formats of GPS and multi-GNSS records. The use of the new TOMION model allows us to take into account a higher number of IPPs and their associated VTEC on the GIM. This is illustrated through the blue crosses and red circles in the map in Fig. 3.

In the experiment of this section, we compare the interpolation quality of the ADIGIM (dictionary of GPS-only measures) and Kriging, using in both cases the IPPs derived from the multi-GNSS. For ease of comparison the structure of Table 2 is used in the assessments for this section in Tables 4 and 5. Also, the comparison in this section is done on the day where there was the loss of stations event in the legacy computation of UQRG, (i.e., more than 100 stations lost on day 326 of 2019), in order also to show two dimensions of the robustness, i.e., loss of stations, and the use of multi-GNSS. 
Table 4 GIM assessment

comparing to JASON3 VTEC, at 20-degree latitudinal bands (in TECUs), with the GPS data in a flexible format

Table 5 GIM assessment comparing to JASON3 VTEC, at 20-degree latitudinal bands (in TECUs), with the multi-GNSS data in a flexible format

\begin{tabular}{|c|c|c|c|c|c|c|c|c|c|c|}
\hline \multirow[t]{3}{*}{ Lat $\left({ }^{\circ}\right)$} & \multicolumn{8}{|c|}{ With RINEX v3 GPS archive } & \multirow{2}{*}{\multicolumn{2}{|c|}{ JASON3 }} \\
\hline & \multicolumn{4}{|c|}{ Kriging } & \multicolumn{4}{|c|}{ ADIGIM } & & \\
\hline & $\overline{\text { Bias }}$ & SD & RMS & ERR\% & $\overline{\text { Bias }}$ & SD & RMS & ERR\% & $\overline{\text { VTEC }}$ & \#OBS \\
\hline $50-70$ & -0.77 & 1.99 & 2.13 & 54.6 & -0.40 & 1.80 & 1.85 & 47.3 & 3.90 & 2771 \\
\hline $30-50$ & 1.84 & 1.78 & 2.56 & 35.0 & -0.77 & 1.89 & 2.04 & 27.8 & 7.33 & 4616 \\
\hline $10-30$ & -2.56 & 2.23 & 3.39 & 34.1 & 2.49 & 3.69 & 9.96 & 37.0 & 9.96 & 5745 \\
\hline-10 to 10 & -2.23 & 2.68 & 3.49 & 24.7 & -2.42 & 2.32 & 3.36 & 23.8 & 14.11 & 7034 \\
\hline-30 to -10 & -2.38 & 2.32 & 3.32 & 24.7 & -2.48 & 2.53 & 3.54 & 26.3 & 13.46 & 7557 \\
\hline-50 to -30 & -1.59 & 2.08 & 2.62 & 28.6 & -1.54 & 1.97 & 2.50 & 27.3 & 9.16 & 9880 \\
\hline-70 to -50 & -0.72 & 2.68 & 2.77 & 29.1 & 0.28 & 2.80 & 2.81 & 29.5 & 9.54 & 9070 \\
\hline
\end{tabular}

Left column experimental new TOMION model plus Kriging. Center: ADIGIM Right: Jason3 reference, on day 326 of 2019

\begin{tabular}{|c|c|c|c|c|c|c|c|c|c|c|}
\hline \multirow[t]{3}{*}{ Lat $\left(^{\circ}\right)$} & \multicolumn{8}{|c|}{ With RINEX v3 Multi-GNSS archive } & \multirow{2}{*}{\multicolumn{2}{|c|}{ JASON3 }} \\
\hline & \multicolumn{4}{|c|}{ Kriging } & \multicolumn{4}{|c|}{ ADIGIM } & & \\
\hline & $\overline{\text { Bias }}$ & SD & RMS & ERR\% & $\overline{\text { Bias }}$ & SD & RMS & ERR\% & $\overline{\mathrm{VTEC}}$ & \#OBS \\
\hline $50-70$ & -0.67 & 2.18 & 2.28 & 58.4 & 0.24 & 1.80 & 1.81 & 46.5 & 3.90 & 2771 \\
\hline $30-50$ & -1.69 & 1.84 & 2.50 & 34.1 & -0.18 & 1.99 & 2.00 & 27.3 & 7.33 & 4616 \\
\hline $10-30$ & -2.12 & 2.44 & 3.24 & 32.5 & -2.24 & 2.73 & 3.53 & 35.4 & 9.96 & 5745 \\
\hline-10 to 10 & -1.93 & 2.86 & 3.45 & 24.4 & -2.08 & 2.42 & 3.19 & 22.6 & 14.11 & 7034 \\
\hline-30 to -10 & -1.99 & 2.59 & 3.27 & 24.3 & -1.97 & 2.70 & 3.34 & 24.8 & 13.46 & 7557 \\
\hline-50 to -30 & -0.91 & 2.35 & 2.52 & 27.5 & -0.98 & 2.03 & 2.25 & 24.6 & 9.16 & 9880 \\
\hline-70 to -50 & -0.34 & 2.56 & 2.58 & 27.0 & 0.22 & 2.59 & 2.60 & 27.3 & 9.54 & 9070 \\
\hline
\end{tabular}

Left column experimental new TOMION model plus Kriging. Center: ADIGIM. Right: Jason3 reference, on day 326 of 2019
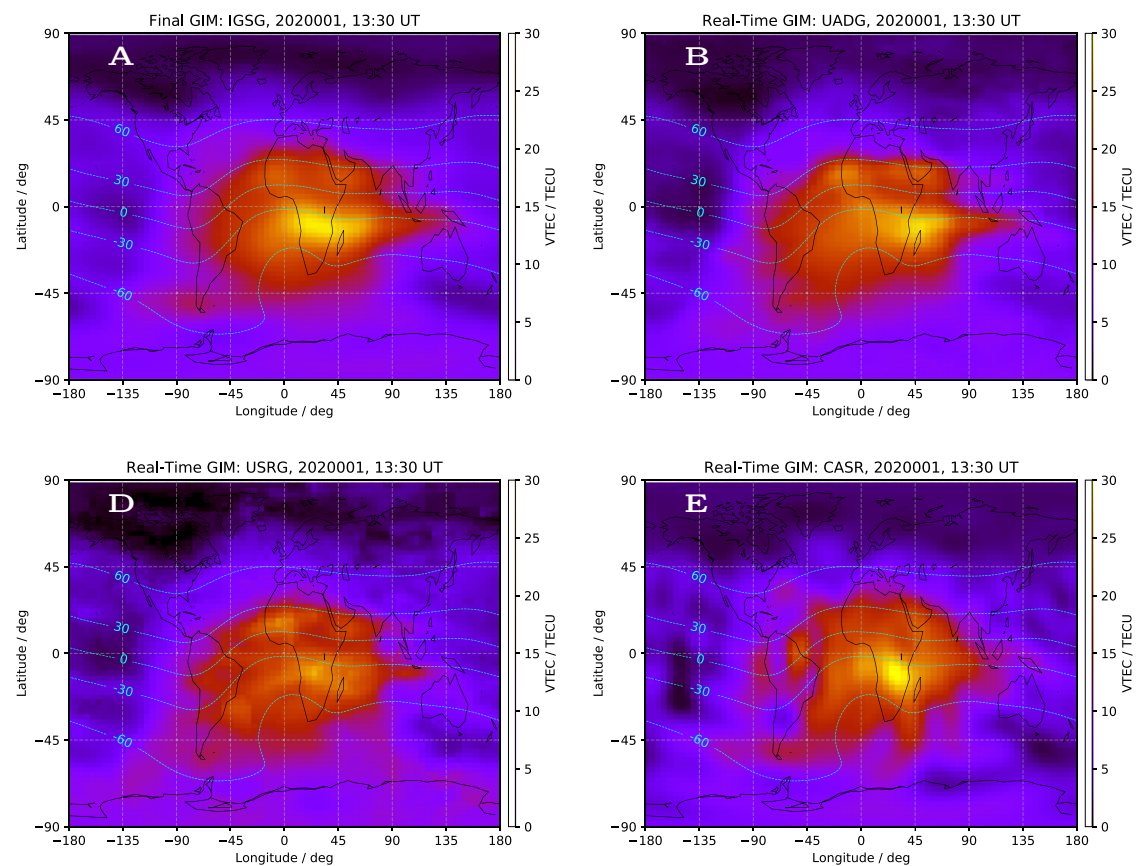

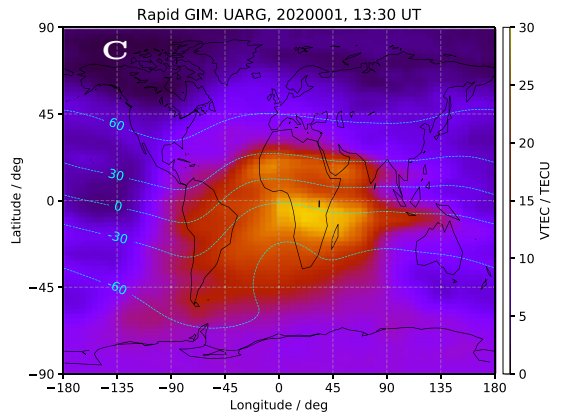

Real-Time GIM: CLK9, 2020001, 13:30 UT

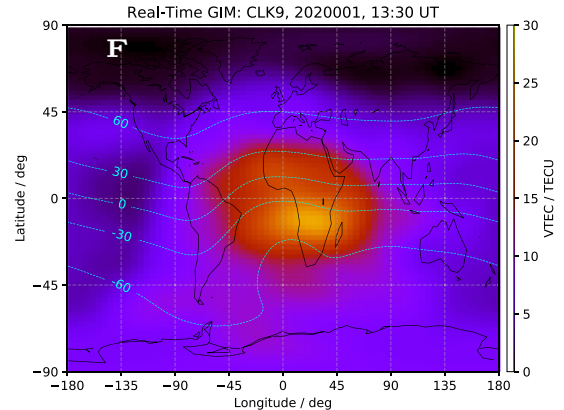

Fig. 4 Example of GIM products at 13:30UT, on the day 1 of 2020. a IGS final GIM IGSG, b UPC experimental RT-GIM UADG, c UPC experimental rapid GIM UARG, $\mathbf{d}$ UPC current RT-GIM USRG, e CAS RT-GIM CASR and f CNES RT-GIM CLK9 
The tables show the interpolation performance by Kriging with the two new GIMs models (flexible GPS-only Table 4 and multi-GNSS Table 5). The method shows a relative error smaller than $40 \%$ in comparison with the legacy GPS-only. This comparison is done for the south high latitudinal region when the loss of stations has been fixed. For the same fixed GPS-only or multi-GNSS data, both the ADIGIM results show slightly better quality than Kriging. The number of obtained IPPs increases as a result of the shift from the legacy TOMION to the experimental new TOMION model, of which the three corresponding GIMs by ADIGIM exhibit increasingly better quality, despite the much smaller improvement than Kriging.

Compared with the VTEC data processed by GPS legacy, GPS RINEX version 3, and multi-GNSS RINEX version 3 approaches, ADIGIM can improve any of the three Kriging results of some southern high latitude regions for the arbitrarily day, in spite of which applies the historical database of legacy Kriging GIMs for the interpolation. Note that the result related to the experimental new TOMION model is just an advance of more consolidated results in this aspect that might be obtained in the future, after extending the entire function of multi-GNSS processing.

\section{Remarks on real-time GIM computation of ADIGIM}

Since the middle of 2019, ADIGIM interpolation has been implemented in the beta test phase for RT-GIM computation, which we denote as the UPC ADIGIM RT-GIM, hereafter UADG, and is an evolution of the current UPC RT-GIM products URTG and USRG (i.e., interpolation done by Kriging and spherical harmonics). The UADG provides the RT-GIM with a temporal resolution of 15 min that is the same with current RT-GIM products, see the characteristics summarized in Table 3. The product is based on the tomography approach of the UPC RT-TOMION model and the interpolation done by the ADIGIM algorithm.

The current UPC rapid GIM UQRG was validated by the assessment in Roma-Dollase et al. (2018) as the likely best quality of GNSS-sounding IGS GIMs for a period of more than one solar cycle. The UPC rapid GIM UQRG was used for constructing the dictionary used for ADIGIM computation in real time. The time interval covered by the UQRG data is from 1996 to the present. This time frame provided examples of GIMs covering ionospheric weather variability spanning more than two solar cycles. The global VTEC observation processed by the UPC RT-TOMION model is the data input for the UADG product. This data flow is the common input for the current RT products, URTG and USRG. The interpolation of UADG is performed by solving the convex optimization problem of approximation of VTEC data from the sparse and

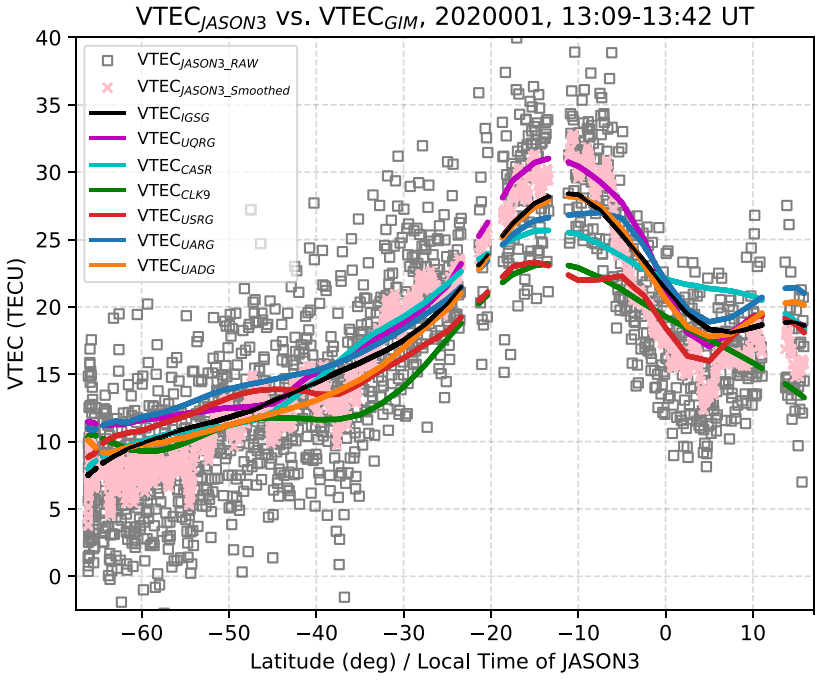

Fig. 5 JASON3 VTEC altimeter measurements (both raw and smoothed observations) versus GIM VTEC, along the JASON3 trajectory during 13:09-13:42UT, on the 1st day of 2020 , from $-70^{\circ}$ to $15^{\circ}$ latitude

non-uniform distribution of global GNSS stations with the dictionary of past maps.

Also, to test the UPC Rapid GIM interpolated by ADIGIM with $\sim 1$-day latency, the UARG, is used to compare, evaluate, and validate the performance of the ADIGIM algorithm in a post-processing scenario, feeding VTEC data from the same IPPs as the post-processed UQRG. As with UADG, UARG likewise uses all UQRG GIMs as historical GIM datasets.

\section{Comparison of real-time GIM products}

In this section, we will compare the performance of different RT-GIM products with the JASON3 benchmark. The RTGIM products for evaluation and their principal features are listed in Table 3. The comparison between all products will be done both visually on given day as in Fig. 4, and by latitude as in Fig. 5.

In Fig. 4, we show the GIMs interpolated by ADIGIM for a given day, e.g., day 1 of 2020, as well as the maps provided by other IGS analysis centers. In the upper left of the Figure, we show the IGS final GIM (IGSG). The IGSG is constructed as the weighted mean of several IAACs' final GIMs and has been shown to perform better quality than other individual final ones (Hernández-Pajares et al. 2009). Even if the latency is of more than 10 days, the IGSG is taken as the benchmark reference for comparing the performance of the RT-GIM estimation methods. An example is shown in the snapshot at 13:30 UT in Fig. 4A (upper left) is the linear interpolation from the product with $2 \mathrm{~h}$ resolution Schaer et al. (1998). The result of applying the method of 
this work, i.e., RT-GIM UADG is shown in Fig. 4B (upper middle). This is the GIM by using ADIGIM for interpolating the RT VTEC data in the interval 13:15-13:30 UT (see locations and associated intensity in Fig. 1B), in addition the ADIGIM for the post-processed GIM UARG is shown in Fig. 4C (upper right). For comparison purposes in Fig. 4D-F, we show, respectively, the current RT-GIM products of UPC (USRG), CAS (CASR), and CNES (CLK9). Note that all of them use the interpolation approach based on spherical harmonics and their extensions in addition to their respective GNSS RT-processing models.

The UADG estimate is more in agreement with the IGSG estimate than the other RT-GIMs estimates. As we do not have the ground truth, we will take the IGSG product as a benchmark. Notably, in terms of the smooth transition from high VTEC regions to other regions, which is compatible with very quiet geomagnetic activity (see the planetary 3-h-range index $k p \leq 1$ corresponding to this time in (GFZ-Postdam 2020)). The comparative improvement of the UADG concerning other methods is reflected in a greater similarity in the general morphology, especially in the regions of higher ionization, and the distinct two ionospheric peaks from the map. Furthermore, the location of peaks and their fading tails along about $\pm 30^{\circ}$ geomagnetic latitudes appear clearly in the RT-GIM UADG, and the final GIM IGSG also clearly shows the delayed response to the ionospheric fountain effect. They also exhibit the peak present at most GIMs at southern high latitudes that might be related to the Weddell sea anomaly of southern summer.

In addition, there is the interpolation region with high uncertainty at the south-eastward peak tail with low VTEC intensity over the south Indian ocean, far away from the ground GNSS observation (see Fig. 1). The distortion due to this uncertainty appears flat in the UADG, whereas a distortion appears as a branch in the USRG by spherical harmonics in Fig. 4D. Note that the small differences in the above-mentioned tails between UADG and UARG indicate the compatible characteristics, despite the high dependence on the quality and the coverage of RT/postponed VTEC map.

Next, as a complement to the Global assessment with IGSG, we will assess the trajectory of the JASON3 satellite and also compare the RT results with the post-process product. In Fig. 1A (orange dots), we show the trajectory of the JASON3 satellite in the time interval 13:09-13:42 UT. During this time interval of about $\sim 30 \mathrm{~min}$, the JASON3 satellite measured the VTEC as it moved from the south polar to the north low latitude region, i.e., from $-70^{\circ}$ to $15^{\circ}$. The comparison of the performance of the different RT products is shown in Fig. 5 where we overlap the VTEC values of the different RT-GIM interpolation products along the corresponding section of the GIM maps. That is the section of the trajectory over the maps shown in Fig. 4 measured at that moment. This allows us to compare the different RT VTEC estimations from the GIM products with the JASON3 measurements.

In Fig. 5, the JASON3 VTEC measurements are marked in gray (raw values) and in pink (smoothed values). As one can see from Fig. 5, the VTECs values corresponding to the benchmark reference IGSG (in black) and UPC rapid UQRG (in magenta) are the curves that best approximate the JASON3 VTEC measurements, being the UQRG slightly better. Next are both the post-processed UARG (in blue) and the RT UADG (in yellow) (both based on the ADIGIM) following, very closely both the two peaks at the equatorial region and also the VTEC trend in the high latitude region. We would like to point out that ADIGIM can have comparable quality with the final IGSG, either working in RT or post-process. On the other hand, the UPC RT-GIM USRG (in red) only shows a good approximation for the spatial variation at local noon at the equatorial region, while CASR (in cyan) and CLK9 (in green) only show the good performance in the high latitude. In this typical example of JASON3 pass, the best behaving GIMs are the ones combining the tomographic modeling (implicitly estimating the mapping function under the two-layer voxel model) with the better interpolation (UQRG, post-processing with Kriging, and UARG and UADG, ADIGIM in post-processing and real-time) or the combination of IGS GIMs including UQRG. This is in agreement with the much better behavior of the voxel-based tomographic model already shown in Figure 2 in Hernández-Pajares et al. (1999).

\section{Performance of the GIMs estimation referenced to JASON3 over 200+ days}

The evaluation of the quality of the interpolation and the comparison between methods is done from the data obtained over the trajectory of the JASON3 satellite. Since the satellite's path is a trajectory over the globe, the evaluation of the quality of the GIMs and the interpolation is not simple. The global VTEC measurement obtained from the JASON3 satellite is determined by the period of the repeated orbit $(\sim 10$ days), and it allows, considering as well previous altimeters, to evaluate the global quality of the GIMs as it was previously done in (Roma-Dollase et al. 2018) during more than one solar cycle.

In this work, the JASON3 VTEC observations we have collected for more than 200 days, i.e., from day 258 of 2019 to day 155 of 2020 . The comparison will be done with all the current GIM products of UPC-IonSAT and other IAACs. The quality of GIMs in a different time and location was determined in the following way: First, we compute the time series the VTEC difference, consisting of JASON3 VTEC minus GIM VTEC on associated JASON3 passes, i.e., $\left(\mathrm{VTEC}_{J A S O N 3}-\mathrm{VTEC}_{\text {GIM }}\right)$. Then, this difference is divided 
into several sub-series consisting of Spatio-temporal cells composed of 2-h time slots times 5-degree latitudinal zones. In order to perform the more details of the latitudinal bias and reflect better the standard deviation of the GIMs, so the smaller latitudinal zones than the ones used than Sect. 5.2. In addition, the time slot of $2 \mathrm{~h}$ is chosen for the approximated period of the circular non-sun-synchronous orbit regarding JASON3. Finally, these sub-series in each cell are assessed, respectively, for the bias and standard deviation.

Note, that in this evaluation we have discarded the days when the UPC RT-GIM service was down for technical reasons. Also, the performance of ADIGIM and other interpolation techniques will be analyzed and discussed. The analysis is done from two points of view:

- The spatio-temporal performance (see Sect. 8.1)

- The performance of the products by latitude. (see Sect. 8.2)

\subsection{Spatio-temporal performance of GIM VTECs}

The performance of ADIGIM will be assessed by comparing it with the final performance of the IGSG, the rapid UQRG, and other RT-GIMs (see details of all the products in Sects. 1 and 6). The assessment will be made referenced to the VTEC values measured by JASON3.

In Figs. 6 and 7, we depict the time series of daily GIMs a) in terms of the latitude (y-axis) vesrus date (x-axis) in the upper rows, with a row per product and b) in the lower row aligned by date with the upper time series the difference VTEC, i.e., $\delta V$ defined as: $\delta \mathrm{V}=\mathrm{VTEC}_{J A S O N 3}-\mathrm{VTEC}_{\text {GIM }}$. This arrangement was decided in order to be able to compare the performance by latitude time aligned with the global performance.

\subsubsection{Bias of VTEC JASON3 $_{3}-$ VTEC $_{G I M}$}

First we analyze the bias of GIM with regard to JASON3, i.e., the VTEC difference $\delta \mathrm{V}=\mathrm{VTEC}_{J A S O N 3}-\mathrm{VTEC}_{\text {GIM }}$. Figure 6A (upper rows) shows the $\delta V$ term of the bias as a time series of daily for the different GIM products. The y-axis aggregates the VTEC by 5 degrees of latitude. The plotted bias is computed as the weighted average of boxes ( 1 day $x$ 5 degrees). For comparison purposes, we plot also the global mean bias per day as a time series in Fig. 6B (lower row). Note the absence of GIM data or JASON3 observation is depicted in way of blanks, and the days of UPC RT-GIM services were down for technical reasons have been discarded.

The time evolution of the post-processed (i.e., non-RT) IGSG and UQRG $\delta V$ bias (6A upper row) shows negative biases from -0.5 TECU in polar regions to -4 TECU near the equator, due to the plasmaspheric VTEC distribution above the JASON3 altimeter $(1300 \mathrm{~km})$ and below the GNSS trans- mitters ( 20,000 km), see Hernández-Pajares et al. (2009). As for the temporal characteristics, in southern latitudes near the pole, both persistently keep a value close to the negative minimum $\sim-0.25$ to -1 TECU during the first 40 days, then drop to less than -3 TECU in the next 100 days, returning to the initial value afterward. At high northern latitudes, there is similar behavior, in this case with a non-negative bias.

Similar to the IGSG, the biases of RT-GIMs such as CASR, CLK9, and USRG exhibit the maximum negative bias values at the equatorial region, while they are mitigated from mid to high latitudes. Note that the two-dimensional distribution consisting of the biases at the Spatio-temporal grids (1 day $\times 5$ degrees of latitude) does not appear to be as smooth as the post-processed ones, and even exhibits a large variation between neighboring grids. In particular, CLK9 shows some of the largest positive biases at low latitudes differing from other GIMs. The daily time series of the spatial mean of the bias (Fig. 6B lower row) shows similar temporal characteristics between post-processed GIMs like IGSG versus UQRG while larger bias for the RT-GIMs.

The two GIM products using the ADIGIM algorithm, i.e., the RT UADG and post-processed UARG, are shown in Fig. 6 the above-mentioned GIMs. The ADIGIM reprocessed UARG shows almost the same distribution of bias as UQRG (same VTEC measurements). That is a negative maximum around the equator and mitigated in other regions. Similarly, the RT UADG with ADIGIM interpolation shows a bias distribution compatible with USRG with common VTEC inputs. Moreover, UADG appears to have less noise and a smoother spatial distribution than other RT-GIMs and is most similar to the post-processed GIMs.

Note that the difference in bias between RT and retrospective GIMs is significant, which may be related to the systematic biases of different ionospheric models (e.g., CLK9 vs. UQRG), see previous research in Orús et al. (2002); Liu et al. (2018), or to the GNSS ionospheric models working in RT or post-processing scenarios (e.g., UQRG vs. USRG), see the comparison and assessment in Li et al. (2020). Besides, shown in Figure 12 and associated comments in Hernández-Pajares et al. (2009), such few TECU of GIM VTEC greater than altimeter VTEC at low latitudes agrees with the expected plasmaspheric electron content at low latitudes in between altimeter and GPS satellites heights.

As can be seen, that ADIGIM preserves the overall VTEC intensity of GIMs. Also, does not induce the local noise of GIMs, and smooths the noisy bias, which has the performance as in post-processed mode.

\subsubsection{Standard deviation of $\mathrm{VTEC}_{J A S O N 3}-\mathrm{VTEC}_{G I M}$}

Next, we analyze the standard deviation of $\delta V$. In the same way as in the figure regarding the bias, in Fig. 7A (upper 
Fig. 6 Daily bias of GIMs regarding

$\mathrm{VTEC}_{J A S O N 3}-\mathrm{VTEC}_{G I M} \cdot \mathbf{a}$ The weighted average of bias when JASON3 locating in the corresponding 5 degree latitudinal zones; $\mathbf{b}$ weighted average of bias for all the latitudinal bins, the color code for different GIM products. For ease of comparison, the rows are aligned by date

Fig. 7 Daily standard deviation (Std.Dev.) of GIMs regarding VTEC $_{\text {JASON3 }}$ - VTEC VIM. $_{\text {a }}$ The weighted average of bias when JASON3 is located in the corresponding 5 degree latitudinal zones; $\mathbf{b}$ weighted average of bias for all the latitudinal bins, the color code different GIM products. For ease of comparison, the rows are aligned by date
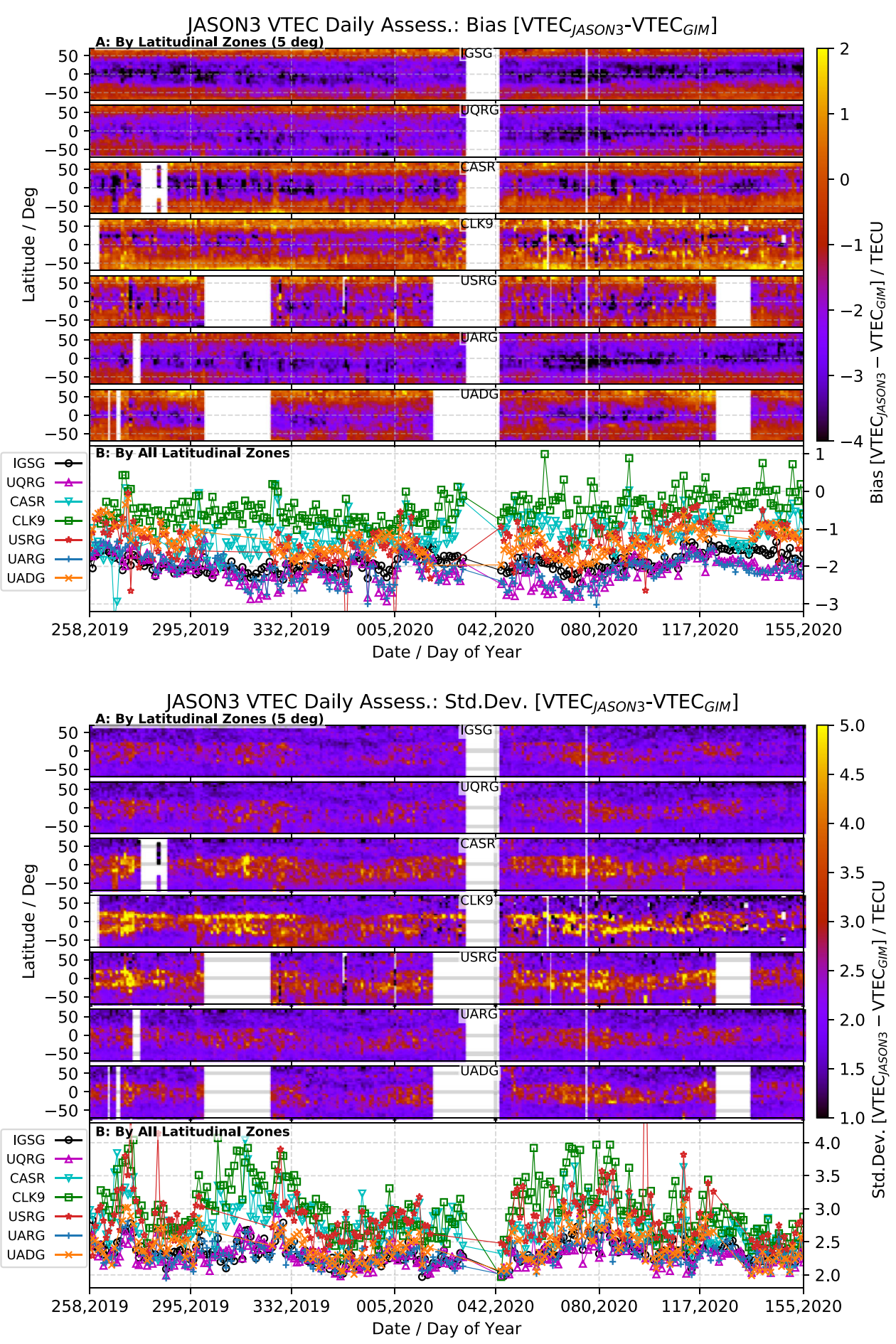

rows), we show the time series of the standard deviation of $\delta V$, in steps of 5 degrees as a function of time. The values in boxes of ( 1 day $\times 5$ degree of latitude) are the mean values of the standard deviation along each latitude interval.

The standard deviations $(\sigma)$ of all GIM products show similar behavior in terms of latitude, i.e., the GIMs at lower latitudes in an absolute sense are of poorer quality than in other regions coinciding with the high VTEC values and gradients associated with the equatorial anomaly peaks. And their time-varying characteristics also coincide in particular, the $\sigma$ shows oscillations over different periods. For instance, two periods from day 265 to 280 of 2019 and from day 70 to 90 of 2020. The retrospective IGSG and UQRG show not only a significantly lower standard deviation compared with the RT-GIMs, but also do not exhibit a significant deterioration in the equatorial region where the two maximum peaks 


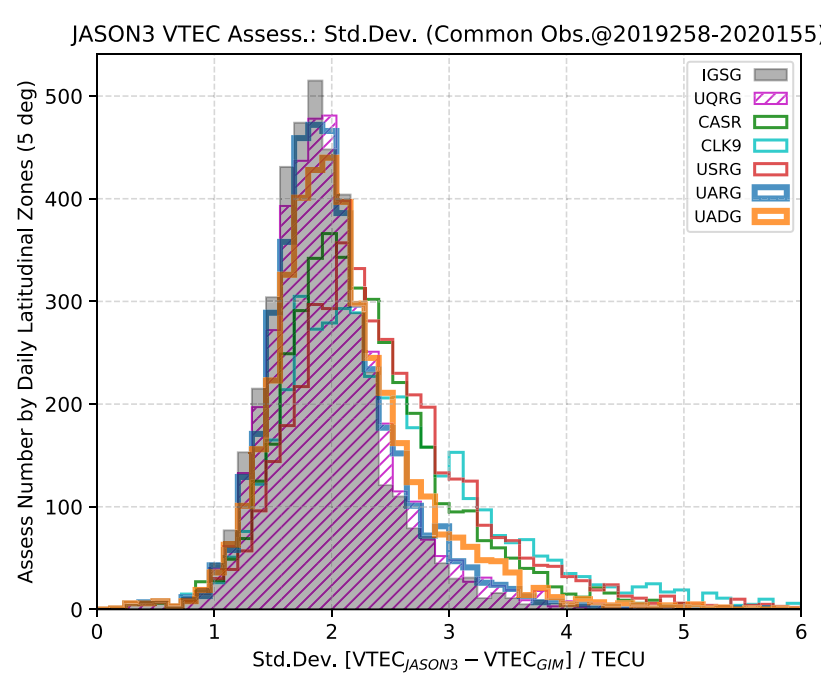

Fig. 8 Histogram of the VTEC standard deviation (Std.Dev.) of the GIMs products referred to the JASON3 observation at Spatio-temporal cells consisting of 1-day duration $\times 5$-degree latitudinal zone, the color code distinguishes the different GIM products

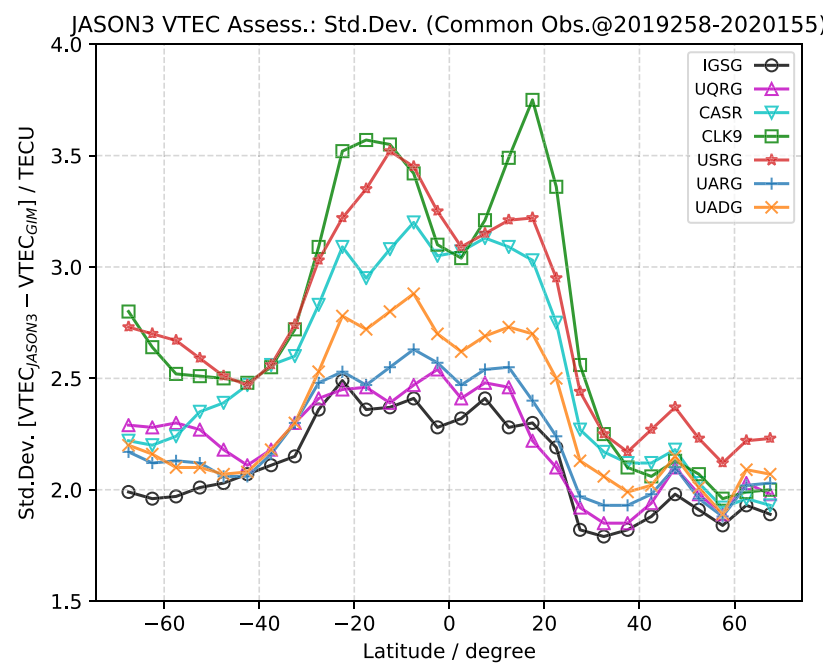

Fig. 9 VTEC standard deviation (Std.Dev.) versus latitudinal zones, the color code representing for different GIMs. Note that the standard deviation is weighted by the number of JASON 3 observations in cells with the same 5-degree intervals of latitude

of the ionosphere normally appear. See the example of different estimation capabilities related to these peaks in Sect. 6. It is expected that the UQRG will have worse performance than the UARG because they have the same input and the UARG is estimated using several decades of UQRG maps. However, the UARG in post process has almost the same performance as the UQRG, and in some cases is better. Also, the standard deviations of UQRG and UARG on the 326th day of 2019 are shown in Fig. 7, are similar to the ones in Table 2, which confirms the evaluating consistency under the two different latitudinal zones.

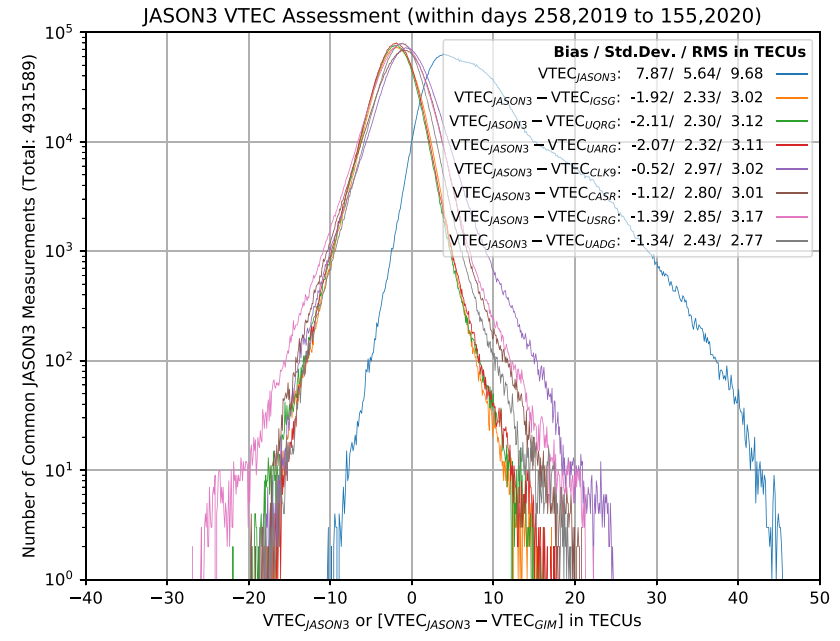

Fig. 10 Histogram, in log scale for the number of counts, of VTEC difference of JASON3 measurement minus GIMs value during day 258 of 2019 to day 155 of 2020 , the color code for different GIMs. The histogram of the reference values of JASON3 is represented in gray. The corresponding overall bias, standard deviation (Std.Dev.), and RMS are indicated in the legend

As for the other interpolation methods, we have the following features related to the performance in terms of standard deviation (see Fig. 7A upper row). The interpolation is done by the products RT CASR and USRG exhibit similar behavior in time and space, with a higher standard deviation than products related to the ADIGIM method, while the CLK9 exhibits a larger standard deviation at the equatorial region. The RT UADG by ADIGIM is of much better quality than other RTGIMs at all latitudes and exhibits significant improvement compared with UPC RT-GIM USRG based on spherical harmonics.

Another approach consists of plotting the time evolution of the mean of the standard deviation taken along the latitude axis (see Fig. 7B lower row). The post-processed GIM UQRG (purple triangle) systematically shows the least standard deviation compared with the other methods, including the combined product IGSG (black circle), while post-processed GIM UARG (blue plus) based on the ADIGIM is better in a small number of days. Finally, the RT-GIM UADG shows the lowest standard deviation in the time series, following the oscillations common to all products (see CASR in a cyan inverted triangle, CLK9 in blue rectangular, and USRG in red star). It also displays in a significant part of the time (> $40 \%$ of days) a standard deviation almost equal to that of the products in post process.

\subsection{Performance of GIM products on common JASON3 passes}

In Sect. 8.1, we examined that the bias and standard performance of the GIM products computed in cells of 1 day $\times 5$ 
Table 6 Summary of GIMs assessment by 4,253,502 common altimeter JASON3 VTEC measurement (in TECUs), for a period of +200 days, from day 258 of 2019 to day 155 of 2020

\begin{tabular}{llllll}
\hline GIM Id & Latency & Bias & SD & RMS & ERR\% \\
\hline IGSG & $>15$ days & -1.93 & $\mathbf{2 . 3 3}$ & 3.03 & 38.5 \\
CODG & $\sim 5$ days & -1.34 & 2.46 & 2.80 & 35.6 \\
JPLG & $\sim 4$ days & -3.34 & 2.45 & 4.15 & 52.7 \\
UPCG & $\sim 15 \mathrm{~h}$ & -2.23 & 2.38 & 3.26 & 41.4 \\
ESAG & $\sim 4$ days & -0.87 & 2.83 & 2.96 & 37.6 \\
WHUG & $\sim 4$ days & -1.39 & 2.52 & 2.87 & 36.5 \\
CASG & $\sim 4$ days & -1.50 & 2.46 & 2.88 & 36.6 \\
EMRG & $\sim 2$ days & -1.69 & 2.61 & 3.11 & 39.5 \\
CORG & $\sim 7 \mathrm{~h}$ & -1.29 & 2.64 & 2.94 & 37.4 \\
JPRG & $\sim 7 \mathrm{~h}$ & -3.44 & 2.48 & 4.24 & 53.9 \\
UQRG & $\sim 16 \mathrm{~h}$ & -2.14 & $\mathbf{2 . 3 0}$ & 3.14 & 39.9 \\
ESRG & $\sim 2 \mathrm{~h}$ & -1.01 & 2.86 & 3.04 & 38.6 \\
WHRG & $\sim 20 \mathrm{~h}$ & -1.35 & 2.55 & 2.89 & 36.6 \\
CARG & $\sim 10 \mathrm{~h}$ & -1.51 & 2.59 & 3.00 & 38.1 \\
EHRG & $\sim 3 \mathrm{~h}$ & -1.07 & 2.69 & 2.90 & 36.8 \\
CASR & RT & -1.14 & 2.80 & 3.02 & 38.4 \\
CLK9 & RT & -0.53 & 2.98 & 3.03 & 38.5 \\
USRG & RT & -1.41 & 2.85 & 3.18 & 40.4 \\
UARG & $\sim 20 \mathrm{~h}$ & -2.09 & $\mathbf{2 . 3 2}$ & 3.13 & 39.7 \\
UADG & RT & -1.34 & $\mathbf{2 . 4 3}$ & 2.77 & 35.1 \\
\hline
\end{tabular}

${ }^{\mathrm{a}}$ Latency of UADG is the delay for RT-GIM for each epoch

degrees of latitude in an interval of 200+ days. In this interval, the best RT-GIM product was the UADG. Next, we show the standard deviation of the GIM estimators as a function of latitude. In this representation a time average is made, so Fig. 9 provides complementary information to Fig. 7. Note that the GIMs were validated on the $\sim 4.93$ million common JASON3 passes in terms of the associated $\delta \mathrm{V}$ time series.

The first analysis consists of computing the histogram of the standard deviation of the $\delta \mathrm{V}$, in boxes of 1 day $\times 5$ degrees of latitude. In Fig. 8, we show the histogram for the interested GIM products. Consisting of 4000 groups of 1 day $\times 5$-degrees boxes measured over the JASON3 trajectories. The post-processed products, IGSG (gray filling) and UQRG (magenta hatched line) show a higher density of observations located at the lower values of the standard deviation, i.e., in the bins of 1.6-2.2 TECU.

Note the counts in the bins below 1.6 TECU show that IGSG is slightly better than UQRG. This phenomenon could be explained because the UQRG in the current UPC TOMION model uses a lower number of GNSS stations. The legacy TOMION model supports limited versions of RINEX. An example is the previously mentioned case of missing stations in Antarctica, which distorted the UQRG quality. Also, it could be explained because the number of IAACs provid- ing final GIMs for combining IGSG has been increased from 2 to 4 since mid- 2019 .

The histograms of the products UQRG and IGSG in Fig. 8 practically overlap. Next in terms of performance are the two products based on the ADIGIM algorithm, the UARG (blue rectangular), which almost overlaps with the UQRG and IGSG products, and the RT UADG (orange rectangular), which has a similar distribution shifted to the right less than 0.2 TECUs and several samples at the position of the maximum similar to the UQRG and IGSG products. The other RT products such as CASR (green rectangular), CLK9 (cyan rectangular), and USRG (red rectangular) have a distribution that is shifted to the right between 0.3 and 0.5 TECUs, and the number of samples at the position of the maximum is lower, which indicates a flatter distribution biased to higher values.

A further way to compare the products is to depict the standard deviation as a function of latitude (in 5-degree intervals) as in Fig. 9. Note that the standard deviation is weighted by the number of JASON3 observations in cells with the same 5degree intervals of latitude. The best performance concerning JASON3 corresponds to IGSG, with UQRG being slightly worse only in the southern region. RT products based on the ADIGIM algorithm have between $10 \%$ and $20 \%$ lower error in the equatorial region than other RT products. In the southern region, products based on the ADIGIM algorithm show good performance despite that the GNSS processing model UPC TOMION lacks enough available GNSS stations.

In Fig. 10, the histogram of the VTEC residual $\delta \mathrm{V}$ on the common JASON3 is shown on a logarithmic scale to enhance the details in the low-density parts of the histogram. This scale in contrast to the linear scale shown in Fig. 8 allows to comparing better the performance in the range of the queues (i.e., regions where the number of samples per bin is much lower than at the mode of the distribution). The first observation related to the figure is that the distributions are asymmetric, and at the queues, the performance of the UARG is almost indistinguishable from the performance of the UQRG and IGSG. At the right side of the distribution of the RT products, the RT UADG is the most similar (in the sense of a lower probability mass for high error) to the post-processed GIM products, UQRG and IGSG. The next in performance is the CASR, followed by the USRG, and finally by the CLK9. This indicates that the probability of a high-value positive error in the UADG is much lower than the other products. For comparison purposes on the figure, there is a summary of the relevant statistics of each product, i.e., bias, standard deviation, and RMS.

For the non-RT products, the overall standard deviation corresponding to each product is 2.33 TECUs for IGSG, 2.30 TECUs for UQRG, and 2.32 TECUs for UARG. On the other hand, the standard deviation of the RT UADG is 2.43 TECUs or about $5 \%$ higher than the non-RT products. 
And the standard deviation values of the other RT products are 2.80 TECUs for CASR, 2.85 TECUs for USRG, and 2.97 TECUs for CLK9, which is about $15 \%$ worse than the UADG

As a final comparison of the performance of the interpolation model ADIGIM, we will make a comparison to almost all the GIM products of IAACs using common JASON3 passes as a reference, see their main characteristics in Table 3. This comparison is presented in Table 6 and shows that the post-processed product UARG behaves in terms of standard deviation and relative error almost the same as the IGSG which is a combination of several final GIM products. And at the same time, it has almost the same performance as another one of the best products, the UQRG. The RT product UADG provides the standard deviation that are better than the other RT products and also in some cases better than rapid or even final products.

On the other hand, the bias represented in Fig. 6, is depending not only on the performance of the GIM (less accurate in RT scenario) but also on the electron content above the altimeter and below the GNSS transmitter (up to a maximum of few TECUs which is achieved at low latitude). Another point that affects the bias is when the mapping function used by one given analysis center is significantly different from the one used by the others (as shown in the assessment of the JPL GIM product (JPLG and JPRG) at Table 6). However, the standard deviation of JASON VTEC versus GIM VTEC is mostly affected by the performance of the GIM, as it was demonstrated in Hernández-Pajares et al. (2017), compared with independent dSTEC assessment over islands. This is the reason such standard deviation values are emphasized in bold in Table 6. And it can be seen that the standard deviation of UARG (computed under rapid latency) is lower than the one for UADG (computed in real-time). Note that the relative error of the UADG is lower than that of the UARG, this is explained that the relative error contains the bias, which is less realistic in the RT TOMION implementation (e.g., the carrier phase ambiguity is not so well determined, see an associated VTEC noise at Fig. 1B), but also affected by the other mentioned factors.

In summary, the metrics evaluated in Table 6 confirm the good performance of ADIGIM, both in RT and non-RT scenarios.

\section{Conclusions}

In this paper, we have introduced a new method for generating GIMs from nonuniform IPP measurements. The method is based on using a dictionary of high-quality past maps and combining a small sample using as a criterion a distance between the current IPP measurements and the VTEC values in the dictionary of past maps at the same coordinates of the IPPs. Also, a regularization term is introduced to guarantee that a small subset of past maps is used.

We have compared the performance of the new interpolation algorithm with a set of current GIM products and with the VTEC measurements from the JASON3 satellite. The assessment has shown that the new product outperforms the other products and in real-time provides GIMs with a quality almost as good as the delayed products that use a higher number of ground stations and better processed data. This improvement was shown in different scenarios, which take into account the measurements over the Indian ocean, where there are no deployed GNSS stations, and with 200 days of JASON3 measurements. Also, we have explored the robustness of the current method regarding the event of losing stations in the polar regions and have shown that the degradation (using JASON3 measurements as reference) was very small.

Acknowledgements This work has been partially supported by the Project PID2019-107579RB-I00 (MICINN). Also was partially supported by the 2017 SGR-0851 Grant of the Generalitat de Catalunya and by the EU Project 101007599 - PITHIA-NRF. The authors acknowledge the GIM products of the IGS and the ionosphere associated analysis centers. The main code of this project was done in Python using the machine learning library scikit-learn.

Author Contributions The mathematical background was worked by HY, EM, and MHP. The experiments were done by HY. The writing of the paper was done by HY and EM. DRD provided the multiGNSS part of the data.

Data availability The experimental UADG and UARG are archived in format of IONEX format, which are open accessible from UPC HTTP site (available at http://chapman.upc.es/tomion/real-time/quick/ archive.uadg/). The data for this paper are available and they can be requested from any of the authors in particular from Heng Yang (h.yang@upc.edu) and Enric Monte Moreno (enric.monte@upc.edu).

\section{Declarations}

Conflict of interest The authors declare that they have no conflict of interest.

\section{References}

Balan N, Bailey G, Su Y (1996) Variations of the ionosphere and related solar fluxes during solar cycles 21 and 22. Adv Space Res 18(3):11-14

Caissy M, Agrotis L (2011) Real-time working group and real-time pilot project. Int GNSS Serv Tech Rep 2011:183-190

Chen SS, Donoho DL, Saunders MA (2001) Atomic decomposition by basis pursuit. SIAM Rev 43(1):129-159 
Dmitriev A, Suvorova A, Klimenko M, Klimenko V, Ratovsky K, Rakhmatulin R, Parkhomov V (2017) Predictable and unpredictable ionospheric disturbances during St. Patrick's day magnetic storms of 2013 and 2015 and on 8-9 March 2008. J Geophys Res Space Phys 122(2):2398-2423

Dow JM, Neilan RE, Rizos C (2009) The international GNSS service in a changing landscape of global navigation satellite systems. J Geod 83(3-4):191-198

Feltens J (2007) Development of a new three-dimensional mathematical ionosphere model at European Space Agency/European Space Operations Centre. Space Weather 5(12):1-17

Feltens J, Angling M, Jackson-Booth N, Jakowski N, Hoque M, Hernández-Pajares M, Aragón-Àngel A, Orús R, Zandbergen R (2011) Comparative testing of four ionospheric models driven with GPS measurements. Radio Sci 46(06):1-11

Forbes JM, Palo SE, Zhang X (2000) Variability of the ionosphere. J Atmos Solar Terr Phys 62(8):685-693

GFZ-Postdam (2020) Indices of global geomagnetic activity. ftp://ftp.gfz-potsdam.de/pub/home/obs/kp-ap/tab/. Accessed 1 Aug 2020

Ghoddousi-Fard R (2014) Gps ionospheric mapping at natural resources Canada. In: IGS workshop, Pasadena

Hastie T, Tibshirani R, Wainwright M (2015) Statistical learning with sparsity: the lasso and generalizations. CRC Press, Boca Raton

Hernández-Pajares M (2014) Inputs received from Ionospheric research groups on activities related with RT global electron content determination. IGS RT WG splinter meeting, June 23. Pasadena, CA, USA, pp 1-15

Hernández-Pajares M, Roma-Dollase D (2017) Examples of igs realtime ionospheric information benefits: space weather monitoring, precise farming and rt-gims. In: IGS workshop, pp 3-7

Hernández-Pajares M, Juan J, Sanz J (1999) New approaches in global ionospheric determination using ground GPS data. J Atmos Solar Terr Phys 61(16):1237-1247

Hernández-Pajares M, Juan J, Sanz J, Colombo OL (2000) Application of ionospheric tomography to real-time GPS carrier-phase ambiguities resolution, at scales of $400-1000 \mathrm{~km}$ and with high geomagnetic activity. Geophys Res Lett 27(13):2009-2012

Hernández-Pajares M, Juan J, Sanz J, Orus R, Garcia-Rigo A, Feltens J, Komjathy A, Schaer S, Krankowski A (2009) The IGS VTEC maps: a reliable source of ionospheric information since 1998. J Geod 83(3-4):263-275

Hernández-Pajares M, Roma Dollase D, Krankowski A, García Rigo A, Orús Pérez R (2016) Comparing performances of seven different global VTEC ionospheric models in the IGS context. In: International GNSS service workshop (IGS 2016), Feb 8-12, 2016, Sydney, Australia, pp 1-13

Hernández-Pajares M, Roma-Dollase D, Krankowski A, García-Rigo A, Orús-Pérez R (2017) Methodology and consistency of slant and vertical assessments for ionospheric electron content models. J Geod 91(12):1405-1414

Hernández-Pajares M, Roma-Dollase D, Garcia-Fernàndez M, OrusPerez R, García-Rigo A (2018) Precise ionospheric electron content monitoring from single-frequency GPS receivers. GPS Solut 22(4): 102

Jakowski N, Mayer C, Hoque M, Wilken V (2011) Total electron content models and their use in ionosphere monitoring. Radio Sci 46(6)

Laurichesse D, Blot A (2015) New CNES real time products including BeiDou. In: IGS Mail 7183, Nov. 10, 2015

Li Z, Yuan Y, Wang N, Hernandez-Pajares M, Huo X (2015) SHPTS: towards a new method for generating precise global ionospheric TEC map based on spherical harmonic and generalized trigonometric series functions. J Geod 89(4):331-345

Li Z, Wang N, Hernández-Pajares M, Yuan Y, Krankowski A, Liu A, Zha J, García-Rigo A, Roma-Dollase D, Yang H, Laurichesse D,
Blot A (2020) Experimental IGS real-time global ionospheric total electron content modeling. J Geod

Lin JW (2010) Two-dimensional ionospheric total electron content map (TEC) seismo-ionospheric anomalies through image processing using principal component analysis. Adv Space Res 45(11):13011310

Liu L, Yao Y, Kong J, Shan L (2018) Plasmaspheric electron content inferred from residuals between gnss-derived and topex/jason vertical tec data. Remote Sens 10(4):621

Mannucci A, Wilson B, Yuan D, Ho C, Lindqwister U, Runge T (1998) A global mapping technique for GPS-derived ionospheric total electron content measurements. Radio Sci 33(3):565-582

Orús R, Hernández-Pajares M, Juan J, Sanz J, Garcı-Fernández M (2002) Performance of different TEC models to provide GPS ionospheric corrections. J Atmos Solar Terr Phys 64(18):2055-2062

Orús R, Hernández-Pajares M, Juan J, Sanz J (2005) Improvement of global ionospheric VTEC maps by using kriging interpolation technique. J Atmos Solar Terr Phys 67(16):1598-1609

Ren X, Chen J, Li X, Zhang X, Freeshah M (2019) Performance evaluation of real-time global ionospheric maps provided by different igs analysis centers. GPS Solut 23(4):113

Roma Dollase D, López Cama JM, Hernández Pajares M, García Rigo A (2015) Real-time Global Ionospheric modelling from GNSS data with RT-TOMION model. In: 5th international colloquium scientific and fundamental aspects of the Galileo Programme, Oct 27-29 2015, Braunschweig, Germany

Roma-Dollase D, Hernández-Pajares M, Krankowski A, Kotulak K, Ghoddousi-Fard R, Yuan Y, Li Z, Zhang H, Shi C, Wang C, Feltens J, Vergados P, Komjathy A, Schaer S, García-Rigo A, GómezCama JM (2018) Consistency of seven different GNSS global ionospheric mapping techniques during one solar cycle. J Geod 92(6):691-706

Salinas A, Toledo-Redondo S, Navarro E, Fornieles-Callejón J, Portí J (2016) Solar storm effects during Saint Patrick's days in 2013 and 2015 on the Schumann resonances measured by the elf station at sierra nevada (spain). J Geophys Res Space Phys 121(12):12-234

Schaer S (1999) Mapping and predicting the Earth's ionosphere using the Global Positioning System, vol 59. Technische Hochschule Zürich, Institut für Geodäsie und Photogrammetrie, Eidg

Schaer S, Beutler G, Rothacher M, Springer TA (1996) Daily global ionosphere maps based on GPS carrier phase data routinely produced by the CODE analysis center. In: Proceedings of the IGS analysis center workshop 1996

Schaer S, Gurtner W, Feltens J (1998) Ionex: the ionosphere map exchange format version 1. In: Proceedings of the IGS AC workshop, Darmstadt, Germany, vol 9

Solomon SC, Qian L, Burns AG (2013) The anomalous ionosphere between solar cycles 23 and 24. J Geophys Res Space Phys 118(10):6524-6535

Thébault E, Finlay CC, Beggan CD, Alken P, Aubert J, Barrois O, Bertrand F, Bondar T, Boness A, Brocco L et al (2015) International geomagnetic reference field: the 12th generation. Earth Planets Space 67(1):79

Wang N, Li Z (2018) Benefits of IGS RTS for real time ionospheric space weather monitoring. In: IGS Workshop 2018, Oct 29-Nov. 2, Wuhan, China

Weber G, Dettmering D, Gebhard H (2005) Networked transport of rtcm via internet protocol (ntrip). In: A window on the future of geodesy, Springer, pp 60-64

Wright J, Yang AY, Ganesh A, Sastry SS, Ma Y (2008) Robust face recognition via sparse representation. IEEE Trans Pattern Anal Mach Intell 31(2):210-227

Wright J, Ma Y, Mairal J, Sapiro G, Huang TS, Yan S (2010) Sparse representation for computer vision and pattern recognition. Proc IEEE 98(6):1031-1044 
Yang H (2019) On the detection of ionospheric waves, relationship with earthquakes and tsunamis. $\mathrm{PhD}$ dissertation, Universitat Politècnica de Catalunya, Spain. http://hdl.handle.net/2117/166164

Yang H, Monte-Moreno E, Hernández-Pajares M (2017) Multi-TID detection and characterization in a dense Global Navigation Satellite System receiver network. J Geophys Res Space Phys 122(9):9554-9575. https://doi.org/10.1002/2017JA023988
Zhang H, Xu P, Han W, Ge M, Shi C (2013) Eliminating negative VTEC in global ionosphere maps using inequality-constrained least squares. Adv Space Res 51(6):988-1000 